\title{
LA FLORA VASCOLARE DELLA PENISOLA DEL SINIS (SARDEGNA OCCIDENTALE)
}

\author{
Giuseppe FENU \& Gianluigi BACCHETTA*
}

\author{
Centro Conservazione Biodiversità (CCB). Dipartimento di Scienze Botaniche. \\ Università degli Studi di Cagliari v.le Sant'Ignazio da Laconi, 13. 09123 \\ Cagliari (Italia) \\ *Corresponding author: bacchet@unica.it
}

Recibido el 17 de noviembre de 2007, aceptado para su publicación el 18 de febrero de 2008 Publicado "on line" en marzo de 2008

RIASSUNTO. La flora vascolare della Penisola del Sinis (Sardegna Occidentale). Viene presentato lo studio della flora vascolare della Penisola del Sinis; in totale sono state rinvenute 760 unità tassonomiche e in particolare 615 specie, 134 sottospecie, 10 varietà e 1 ibrido, riferibili a 365 generi e 87 famiglie. Le Eudicots sono risultate il gruppo sistematico dominante. Le famiglie più rappresentate sono: Poaceae (99 unità tassonomiche), Fabaceae e Asteraceae (85), Caryophyllaceae (33), Apiaceae (27) e Orchidaceae (24). I generi con maggior numero di taxa sono: Trifolium (19), Silene (14), Limonium e Medicago (13), Ophrys (12), Euphorbia e Vicia (10), Plantago (9), Allium (8) e infine Lotus, Ranunculus e Vulpia (7). Il contingente delle endemiche (54 unità tassonomiche) è risultato pari al $8,97 \%$ della componente mediterranea e mostra una dominanza degli elementi sardo-corsi $(33,33 \%)$ e secondariamente sardi $(24,10 \%)$, i quali unitamente raggiungono il 57,43\% del totale. La flora endemica è costituita da 31 specie, 17 sottospecie e 6 varietà, inquadrati in 38 generi e 22 famiglie. Le famiglie più rappresentate sono risultate le Plumbaginaceae (10), Asteraceae e Lamiaceae (5), Fabaceae (4), Alliaceae, Euphorbiaceae e Ranunculaceae (3); il genere più ricco è risultato Limonium (10), seguito da Allium, Delphinium, Euphorbia, Scrophularia, Silene e Teucrium (2). E'stato elaborato un elenco dei taxa inseriti nelle liste rosse regionali d'Italia, al quale sono state aggiunte tutte le endemiche esclusive e le specie di importanza conservazionistica a livello regionale, per le quali vengono proposte le nuove categorie di protezione secondo i criteri della IUCN. Da tale analisi è emerso che sono presenti 12 unità tassonomiche gravemente minacciate, gruppo che include gran parte delle endemiche esclusive del territorio. Sulla base dei dati morfologici, geologici e bioclimatici relativi ai territori studiati, unitamente alle analisi di tipo floristico ed in particolare della componente endemica e d'interesse fitogeografico, viene proposto un inquadramento biogeografico a livello di sottosettore.

Parole chiave. Flora, diversità vegetale, endemiche, conservazione, biogeografia, Sardegna

ABSTRACT. The vascular flora of Sinis Peninsula (W Sardinia). The vascular flora of Sinis Peninsula (W Sardinia) has been studied; 760 taxa, 615 of which were species, 134 subspecies, 10 varieties and 1 hybrid were found. They were included in 365 genera and 87 families. The Eudicots were dominant over the other systematic groups. The most represented families were: Poaceae (99 taxonomic units), Fabaceae and Asteraceae (85), Caryophyllaceae (33), Apiaceae (27) and Orchidaceae (24). The most represented genera were: Trifolium (19), Silene (14), Limonium and Medicago (13), Ophrys (12), Euphorbia and Vicia (10), Plantago (9), Allium (8) and, finally, Lotus, Ranunculus and Vulpia (7). 
The contingent of endemics (54 taxonomic units) was $8,97 \%$ of the Mediterranean component showing the dominance of Sardinian-Corsican $(33,33 \%)$ and in the second place Sardinian elements $(24,10 \%)$, that together to reach $57,43 \%$ of the total. This flora consisted of 31 species, 17 subspecies and 6 varieties; 38 genera and 22 families were recognized. The most represented families were: Plumbaginaceae (10), Asteraceae and Lamiaceae (5), Fabaceae (4), Alliaceae, Euphorbiaceae and Ranunculaceae (3). The most represented genera were: Limonium (10) and Allium, Delphinium, Euphorbia, Scrophularia, Silene, Teucrium (2). Based on the taxa present in the regional Italian Red List, the species exclusive of Sardinia and important for the conservation has been included. For these species we proposed the news categories of preservation following the IUCN criteria. Our analysis, confirmed the presence of 12 taxa critically endangered as well as this group included the majority of exclusive endemism of the territory. According to the geomorphological, geological and bioclimatic data of the territories studied within the floristic analysis and in particular the endemic and phytogeographical component, we proposed the creation of new biogeographic subsector named Sinisico.

Key words. Flora, plants diversity, endemics, conservation, biogeography, Sardinia

\section{INTRODUZIONE}

Gli studi floristici finora realizzati per la Penisola del Sinis sono costituiti da ricerche condotte tra la metà degli anni 80 e la metà degli anni 90; ad oggi manca uno studio d'insieme di tutta l'area poiché le analisi condotte in passato si sono focalizzate su alcuni settori particolari della fascia costiera: Isola di Mal di Ventre (Mossa et al., 1984), Mari Ermi (Mulas, 1986), Capo Mannu (Bocchieri et al., 1988), Torre Seu (Mulas, 1993) e Capo San Marco (Bocchieri, Mulas, 1996).

Obiettivo della ricerca è stato quello di fornire un elenco floristico completo della fascia costiera e insulare della Penisola del Sinis, escludendo le aree urbanizzate e ponendo come limite quello delle coltivazioni. Tale contributo vuole evidenziare la notevole diversità vegetale presente nell'area determinata da numerosi taxa esclusivi, rari e di rilevante interesse conservazionistico e vuole essere preliminare ad ulteriori ricerche più approfondite al fine di individuare misure gestionali e di conservazione della diversità vegetale dei territori analizzati.

\section{Area di studio}

La Penisola del Sinis (fig. 1) è ubicata nel settore centro-occidentale della Sardegna e considerando l'Isola di Mal di Ventre, oltre allo scoglio del Catalano, ha una superficie complessiva di circa 170 chilometri quadrati e una estensione della linea costiera pari a circa $75 \mathrm{Km}$. L'area oggetto di questo studio ricade amministrativamente nella Provincia di Oristano e, procedendo da nord verso sud, nei comuni di Cuglieri, Narbolia, San Vero Milis, Riola Sardo e Cabras.

Si caratterizza per la morfologia tipicamente sub-pianeggiante con rilievi che, solo sulle pendici basaltiche del Montiferru, tendono ad elevarsi oltre i 200 metri. Tutta l'area è ampiamente utilizzata per le colture agrarie estensive ed intensive e per le attività zootecniche.

La Penisola del Sinis è ubicata all'estremità NW del Graben del Campidano ed è separata dall' area subsidente di Oristano da faglie quaternarie di direzione N-S; queste faglie hanno dislocato le colate neotettoniche basaltiche plio-pleistoceniche, che infatti affiorano a quote di 80 metri, ribassandole di oltre 300 metri (Cherchi et al., 1978). Le litologie affioranti nel territorio possono essere schematicamente raggruppate in cinque unità di diverso significato paleogeografico e strutturale.

Il basamento ercinico che affiora 


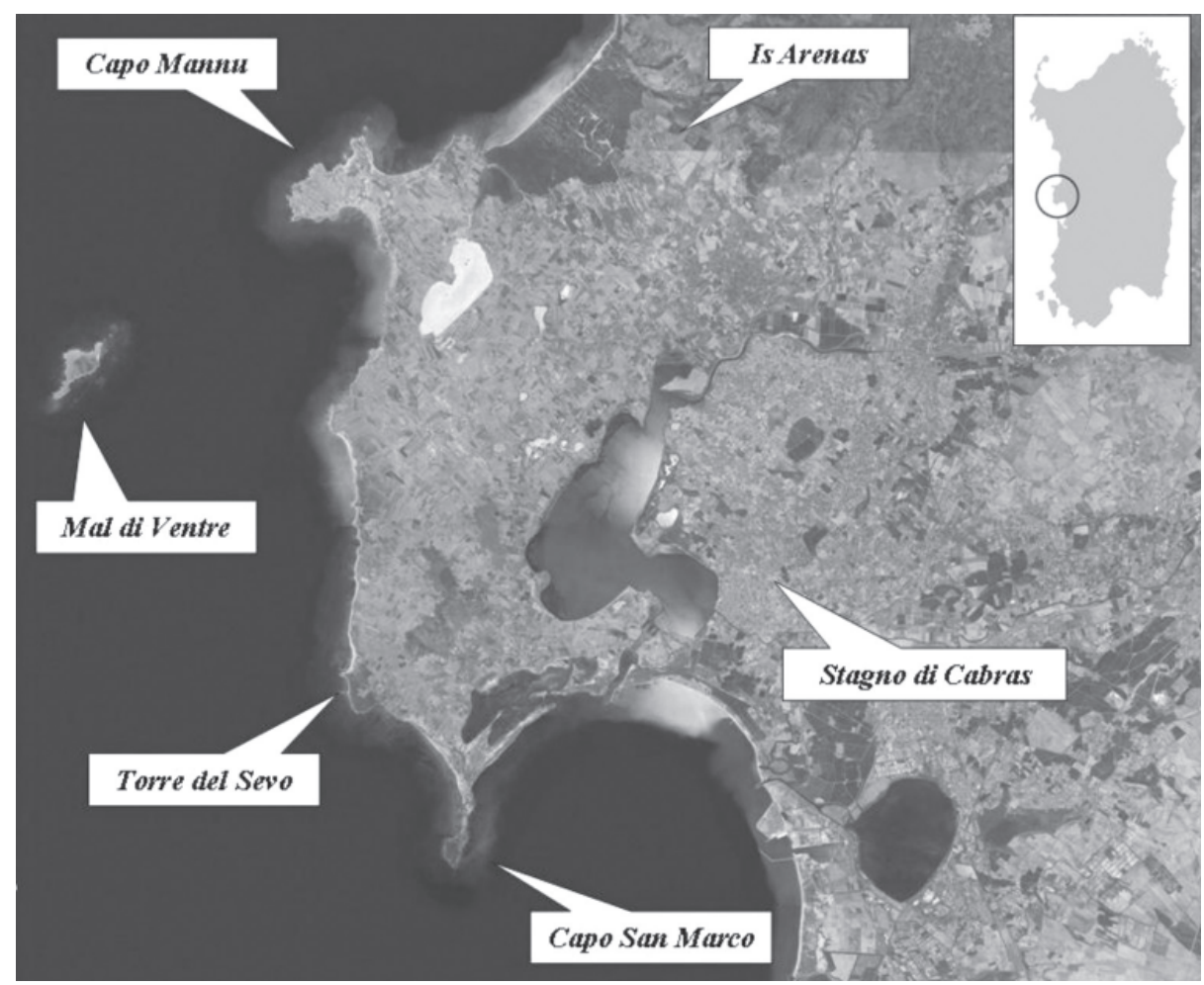

Figura 1. Carta indice dei territori della Penisola del Sinis. Index-map of the territories of Sinis Peninsula.

esclusivamente nell'Isola di Mal di Ventre rappresenta, insieme al promontorio di Capo Pecora, uno dei pochi affioramenti presenti lungo tutto il settore occidentale della Sardegna ed è rappresentato da rocce granitiche a grana medio-grossolana (Deriu, Zerbi, 1964). Su tale basamento poggia il complesso calc-alcalino del Montiferru, che chiude a nord la Penisola del Sinis (Forti, Orrù, 1995).

Il basamento oligo-miocenico inferiore è costituito da andesiti e tufi che affiorano nella parte NE della Penisola del Sinis; su queste vulcaniti poggiano i sedimenti marini del miocene inferiore, costituiti da conglomerati poligenici seguiti da calcari organogeni ad Ostreae; sedimenti di questa fase affiorano anche lungo costa, alla base della falesia occidentale di Capo San Marco (Cherchi et al., op. cit.).

I sedimenti del Miocene superiore affiorano abbondantemente in tutta la Penisola e appartengono al Messiniano e in subordine al Tortoniano; questi ultimi sono costituiti da argille grigio-scure indicanti un ambiente di deposito lagunare, mentre la successione dei depositi messiniani viene suddivisa in tre unità litostratigrafiche che comprendono le argille marnoso-siltose (Formazione di Capo San Marco), seguite da calcari bianchi (calcari laminati del Sinis) e infine da calcari e calcari dolomitici (Formazione di Torre del Sevo).

I sedimenti del Pliocene inferiore sono rappresentati esclusivamente all'estremità di Capo San Marco da breccie costitute da clasti 
messiniani, argille siltose, calcari argillosoarenacei e arenarie argillose (Cherchi et al., op. cit.).

L'ultima unità è rappresentata delle vulcaniti plio-pleistoceniche e dai depositi quaternari; le prime sono rappresentate da colate basaltiche che ricoprono Capo San Marco e lo scoglio del Catalano.

Il Quaternario è costituito principalmente da formazioni eoliche tra le quali si distinguono quelle wurmiane, molto diffuse e composte da arenaria ben cementata con intercalati numerosi livelli di paleosuoli argillosi di colore rosso bruno, che nell'insieme vanno a costituire la Formazione di Capo Mannu (Cherchi et al., op. cit.).

Da segnalare la presenza della panchina organogena tirreniana che affiora lungo tutta la fascia costiera, cui seguono arenarie organogene e le eolianiti a laminazione incrocciata di Su Tingiosu.

I depositi attuali sono costituiti da sabbie di spiaggia e sabbie eoliche sulle coste e da limi e argille presso gli stagni e lungo i principali corsi d'acqua (Cherchi et al., op. cit.).

Le coste del Sinis sono esposte all'azione dei venti e del moto ondoso, vengono quindi modellate continuamente dall'azione del mare che demolisce le falesie ma allo stesso tempo accumula grandi quantità di sedimenti nei tratti di costa bassa. Consistenti depositi sedimentari, costituenti sia le spiagge sommerse che quelle emerse, sono presenti nelle località di Is Arenas,
Maimoni e San Giovanni di Sinis.

In particolare merita una citazione il campo dunare di Is Arenas, tra i più estesi della Sardegna, costituito da sedimenti di natura prevalentemente quarzosa e, in subordine, quarzoso-feldspatica. Nella parte a mare antistante è presente una notevole spiaggia sommersa, limitata da affioramenti discontinui di bancate di arenarie, sulle quali si imposta una prateria a Posidonia oceanica (L.) Delile molto estesa e densa (Forti, Orrù, op. cit.).

Altra particolarità è data dal sistema di Capo Mannu, promontorio costituito prevalentemente da coste alte a falesia di natura calcarea miocenica, collegato alla terraferma da un tombolo di arenarie eoliche, che contribuisce a chiudere le zone umide del complesso di Sa Salina Manna.

In generale, per quanto riguarda l'area in esame e in modo particolare per la sua porzione meridionale, si può concludere che gli arenili sono il risultato di una componente silicoclastica grossolana proveniente dall'erosione dell'Isola di Mal di Ventre, e una componente carbonatica biogenica più fine derivante dall'accumulo di detriti prodotti nelle praterie di P. oceanica (De Falco et al., 2003).

Le analisi sulle tendenze evolutive degli arenili, realizzate negli ultimi anni, hanno confermato che sono in atto fenomeni erosivi sulla totalità delle aree costiere, sia perché il litorale è esposto a un idrodinamismo elevato legato a un moto ondoso intenso, sia

\begin{tabular}{lccccccccccccccc}
\hline $\begin{array}{l}\text { Alt (m) } \\
\text { diOsserv. }\end{array}$ & Anni & Temp. & Gen & Feb & Mar & Apr & Mag & Giu & Lug & Ago & Set & Ott & Nov & Dic & Med \\
\hline $\mathbf{1 0}$ & $\mathbf{3 4}$ & $\max$ & 14,6 & 14,7 & 17,3 & 19,7 & 22,9 & 27,4 & 30,0 & 30,6 & 28,5 & 24,0 & 19,3 & 15,6 & 22,0 \\
& & $\min$ & 5,3 & 5,6 & 7,2 & 9,2 & 12,2 & 15,5 & 17,2 & 17,7 & 16,6 & 13,2 & 9,5 & 6,5 & 11,3 \\
& & med & 9,9 & 10,1 & 12,2 & 14,4 & 17,5 & 21,4 & 23,6 & 24,1 & 22,6 & 18,6 & 14,4 & 11,0 & 16,7 \\
\hline
\end{tabular}

Tabella 1. Andamento delle temperature rilevate per la stazione di Santa Giusta. Temperature values from Santa Giusta meteorology station. 
perché non si hanno apporti sedimentari dall'entroterra (De Falco, Piergallini, 2003).

\section{Inquadramento climatico}

Dal punto di vista climatico viene evidenziato come nella Penisola del Sinis non vi siano stazioni meteorologiche per il rilevamento di dati termici e pluviometrici. I dati relativi alle stazioni prossime indicano scarse precipitazioni nel corso dell'anno, concentrate in autunno-inverno, con un lungo periodo di aridità estiva; le temperature medie si mantengono elevate durante tutto l'anno; i mesi più freddi sono gennaio e febbraio, mentre quelli più caldi luglio e agosto (ENEA, 1990).

Per la stazione di Santa Giusta (tab. 1) i dati indicano temperature medie superiori a $20^{\circ} \mathrm{C}$ nei mesi di giugno, luglio, agosto e settembre, mentre la media annua è di $16,7^{\circ} \mathrm{C}$, con una media delle massime di $22^{\circ} \mathrm{C}$ e una media delle minime di $11,3^{\circ} \mathrm{C}$.

La media annua delle precipitazioni registrata per Santa Giusta è di $600 \mathrm{~mm}$, queste risultano distribuite in 75 giorni piovosi e si verificano soprattutto nel periodo ottobre-dicembre, mentre sono del tutto assenti in luglio e agosto. Per la stazione di Riola Sardo si hanno valori medi delle precipitazioni di $665 \mathrm{~mm}$ annui, concentrati in soli 58 giorni; il periodo estivo è caratterizzato da una accentuata e prolungata aridità, con annate nelle quali il periodo secco si prolunga anche per otto mesi. L'andamento mensile delle precipitazioni per le stazioni di Santa Giusta e Riola Sardo viene riportato in tabella 2.

L'area del Sinis, per la configurazione e posizione geografica, è caratterizzata da un'elevata ventosità; i venti dominanti sono quelli provenienti dal IV quadrante (maestrale e ponente), che spesso raggiungono e superano la velocità di $25 \mathrm{~m} /$ $\mathrm{s}$, e in subordine quelli provenienti dal II e III quadrante (scirocco e libeccio).

Sulla base dei dati disponibili e considerando dal punto di vista metodologico quanto proposto da RivasMartínez (1996) e Rivas-Martínez et al. $(1999 ; 2002)$ è possibile per le aree in esame ipotizzare un bioclima Mediterraneo pluvistagionale oceanico, con termotipo termomediterraneo inferiore-superiore e ombrotipo secco inferiore-superiore.

\section{MATERIALI E METODI}

Le informazioni presenti in questo lavoro derivano da una indagine bibliografica e dall'analisi dei campioni d'erbario depositati presso il Dipartimento di Scienze Botaniche di Cagliari (Herbarium $\mathrm{CAG}$ ), integrate con le osservazioni derivanti da numerose escursioni in campo realizzate tra gennaio 2003 e marzo 2007, durante tutte le stagioni dell'anno.

Per le unità tassonomiche a distribuzione mediterraneo-occidentale, sono stati visionati anche i campioni d'erbario conservati presso il Jardì Botanic de Valencia (Herbarium VAL) e l'erbario virtuale delle

\begin{tabular}{lccccccccccccc}
\hline Stazione & Gen & Feb & Mar & Apr & Mag & Giu & Lug & Ago & Set & Ott & Nov & Dic & Med. Annua \\
\hline Santa Giusta & 68 & 57 & 54 & 49 & 34 & 10 & 3 & 10 & 40 & 82 & 90 & 104 & $600 \mathrm{~mm}$ \\
Riola Sardo & 87 & 68 & 63 & 50 & 35 & 13 & 3 & 10 & 42 & 77 & 107 & 110 & $665 \mathrm{~mm}$ \\
\hline
\end{tabular}

Tabella 2. Andamento mensile delle precipitazioni per le stazioni di Santa Giusta e Riola Sardo. Monthly precipitation values from Santa Giusta and Riola Sardo meteorology stations. 
Isole Baleari (http://herbarivirtual.uib.es/cas/ index.html).

Per la nomenclatura delle famiglie delle Angiospermae si è utilizzato l'ordinamento basato sul recente lavoro realizzato dal Angiosperm Phylogeny Group (2003; http:// www.mobot.org/MOBOT/research/APweb/).

Per la nomenclatura tassonomica si è fatto riferimento principalmente a quanto riportato da Conti et al. (2005); sono state inoltre consultate le seguenti opere: MedChecklist (Greuter et al., 1984-89), Flora Europaea (Tutin et al., 1964-80, 1993), Flora d'Italia (Pignatti, 1982), Flora dels Päisos Catalans (Bolòs, Vigo, 1984-2001), Flora Iberica (Castroviejo, 1986-2006), la monografia“"Le piante endemiche della Sardegna” (Arrigoni et al., 1977-1991), quella di Marchetti (2004) per le Pteridofite e i lavori di Bateman et al. (2003) e Delforge (2005) per le Orchidaceae.

Dal punto di vista tassonomico sono stati inoltre utilizzati alcuni recenti contributi per i generi Limonium (Mayer, 1995; Arrigoni, Diana, 1999), Catapodium e Desmazeria (Brullo et al., 2003), Ononis (Angiolini et al., 2004), Pseudorlaya e Orobanche (Banfi et al., 2005) e Silene (Valsecchi, 1995). Per le abbreviazioni degli autori si è seguito Brummitt et Powell (1992).

La forma e sottoforma biologica è stata direttamente verificata in campo, si basa sulla classificazione di Raunkiaer (1934) ed è espressa secondo le sigle di Pignatti ( $o p$. cit.).

Per le forme corologiche, oltre alle monografie utilizzate per la nomenclatura tassonomica, è stata seguita la classificazione proposta da Pignatti (op. cit.), modificata da Brullo et al. (1996) per quanto riguarda i corotipi mediterranei. Per le endemiche si è adottata la nomenclatura proposta da Arrigoni et Di Tommaso (1991), aggiungendo le categorie delle endemiche sardo-sicule (ESS), tirreniche insulari-nordafricane (ETI-NA) e mediterraneo occidentali insulari (EMOI), come proposto da Bacchetta et Pontecorvo (2005). Per quanto riguarda le unità tassonomiche alloctone sono state utilizzate le categorie proposte da Pysek et al. (2003).

Relativamente ai taxa protetti e/o a rischio d'estinzione, sono state anche specificate le diverse categorie utilizzate dalla IUCN (IUCN, 1994, 2001, 2003; Conti et al., 1997; Pignatti et al., 2001; Scoppola, Spampinato, 2005). Sulla base del lavoro di campo, degli studi realizzati e dei dati bibliografici, vengono proposte le modifiche alle categorie di protezione per i taxa endemici esclusivi e per quelli di interesse conservazionistico presenti nella Penisola del Sinis, secondo le indicazioni della IUCN (2001, 2006).

Nell'elenco floristico viene riportata, per ogni unità tassonomica, la forma biologica, la forma corologica e un indice numerico che riassume la rarità in base alla distribuzione geografica, alla specificità ecologica e alla dimensione delle popolazioni locali, secondo quanto proposto da Rabinowitz (1981) e Rabinowitz et al. (1986). In particolare l'indice di rarità è composto da tre fattori che indicano se l'areale di distribuzione è ampio ( $\mathrm{W}=$ wide) o ristretto ( $\mathrm{N}=$ narrow), se la specie ha una alta specificità ecologica $(\mathrm{R}=$ restricted) oppure è presente in diversi habitat ( $\mathrm{B}=$ broad) e infine se la dimensione delle popolazioni locali è grande $(\mathrm{L}=$ somewhere large) oppure sempre di piccole dimensioni ( $\mathrm{S}=$ everywhere small).

Per le specie alloctone viene indicata tra parentesi la regione d'origine. Le unità tassonomiche segnalate precedentemente in letteratura, ma non ritrovate durante le nostre ricerche di campo ed erbario, vengono precedute da un asterisco. Tutte le entità endemiche sono contrassegnate con un doppio asterisco. I campioni d'erbario sono stati depositati presso Herbarium CAG. 


\section{RISULTATI}

ISOETACEAE

Isoëtes histrix Bory. G bulb. Medit.-Atl. NRS.

\section{EQUISETACEAE}

Equisetum ramosissimum Desf. G rhiz. BoreoTrop. WRS.

\section{POLYPODIACEAE}

Polypodium cambricum L. H ros. Euro-Medit. WBS.

\section{ASPLENIACEAE}

Asplenium onopteris L. H ros. Euro-Medit. WBS.

\section{CUPRESSACEAE}

Juniperus oxycedrus L. subsp. macrocarpa (Sibth. et Sm.) Neilr. P caesp. Circum-Medit. WRL.

Juniperus phoenicea L. subsp. turbinata (Guss.) Nyman. P caesp. Medit.-Irano-Turan. WRL.

\section{PINACEAE}

Pinus halepensis Mill. P scap. Nat. (CircumMedit.). WRL.

Pinus pinea L. P scap. Avv. (Circum-Medit.). WRL.

\section{EPHEDRACEAE}

Ephedra distachya L. subsp. distachya. NP. NW Medit. NRL.

\section{LAURACEAE}

Laurus nobilis L. P caesp. Avv. (Euro-Medit.). WBS.

\section{ARACEAE}

Ambrosina bassii L. G rhiz. S Medit. NBS.

Arisarum vulgare Targ. Tozz. G rhiz. CircumMedit. WBL.

**Arum pictum L. $f$. subsp. pictum. G rhiz. Endem. SA-CO. NBL.

Lemna gibba L. I nat. Boreo-Trop. WRL.

\section{JUNCAGINACEAE}

Triglochin bulbosum L. subsp. barrelieri (Loisel.) Rouy. G bulb. Circum-Medit. WRL.

Triglochin laxiflorum Guss. G bulb. CircumMedit. WRL.
POSIDONIACEAE

Posidonia oceanica (L.) Delile. I rad. Medit.-Atl. WRL.

\section{POTAMOGETONACEAE}

Althenia filiformis Petit subsp. filiformis. I rad. W Medit. NBL.

\section{RUPPIACEAE}

Ruppia maritima L. I rad/I nat. Boreo-Trop. WRL.

Ruppia cirrhosa (Petagna) Grande. I rad. BoreoTrop. WBL.

*Ruppia drepanensis Tinéo. T rept. SW Medit.

*Ruppia maritima L. subsp. brachypus (Gay) Love. I rad. Cosmop.

\section{ZOSTERACEAE}

Zostera marina L. I rad. Medit.Atl. WRL.

Zostera noltii Hornem. I rad. Medit.-Atl. WRL.

CYMODOCEACEAE

Cymodocea nodosa (Ucria) Asch. I rad. Medit.Atl. WRL.

\section{ALLIACEAE}

Allium ampeloprasum L. G bulb. Circum-Medit. WRL.

Allium chamaemoly L. subsp. chamaemoly. G bulb. Circum-Medit. WRS.

Allium commutatum Guss. G bulb. CircumMedit. WRS.

**Allium parciflorum Viv. G bulb. Endem. SACO. NRS.

**Allium roseum L. var. insulare Gennari. G bulb. Endem. SA-CO. NBS.

Allium sphaerocephalon L. G bulb. E Medit. WRS.

Allium subhirsutum L. G bulb. Circum-Medit. WRL.

Allium triquetrum $\mathrm{L}$. G bulb. W Medit. WRL.

Narcissus bertolonii Par. G bulb. Circum-Medit. WRS.

Narcissus tazetta L. G bulb. Circum-Medit. WRS.

**Pancratium illyricum L. G bulb. Endem. SACO-AT. NBS.

Pancratium maritimum L. G bulb. CircumMedit. WRL. 


\section{ASPARAGACEAE}

Agave americana L. P caesp. Nat. (C America). WBS.

Asparagus acutifolius L. G rhiz. Circum-Medit. WBL.

Asparagus albus L. Ch frut. W Medit. WBS.

Asparagus aphyllus L. Ch frut. Circum-Medit. WRL.

Asparagus stipularis Forssk. NP. Circum-Medit. WRL.

Charybdis maritima (L.) Speta. G bulb. CircumMedit. WRL.

Charybdis undulata (Desf.) Speta. G bulb. S Medit. NBS.

Loncomelos narbonensis (Torn. in L.) Raf. G bulb. Euro-Medit. WRS.

Muscari comosum (L.) Mill. G bulb. Euro-Medit. WBL.

**Ornithogalum corsicum Jord. et Fourr. G bulb. Endem. SA-CO. NRL.

Ornithogalum umbellatum L. G bulb. CircumMedit. WRS.

**Prospero autumnale (L.) Speta var. corsica (Boullu) Briq. G bulb. Endem. SA-CO. NBS.

Ruscus aculeatus L. Ch frut. Circum-Medit. WRL.

Urginea fugax (Moris) Steinh. G bulb. SW Medit. NBL.

\section{IRIDACEAE}

**Crocus minimus DC. G bulb. Endem. SA-COAT. NBL.

Gladiolus communis L. subsp. byzantinus (Mill.) A.P. Ham. G bulb. Circum-Medit. WBS.

Gynandriris sisyrinchium (L.) Parl. G bulb. Circum-Medit. WBS.

Iris pseudacorus L. G rhiz. Euro-Medit. WRS.

Romulea bulbocodium (L.) Sebast. et Mauri. G bulb. Circum-Medit. WRS.

Romulea columnae Sebast. et Mauri. G bulb. Circum-Medit. WRS.

Romulea ligustica Parl. G bulb. SW Medit. NBS.

**Romulea requienii Parl. G bulb. Endem. SACO (ITC). NRL.

Romulea rollii Parl. G bulb. W Medit. NBS.

\section{ORCHIDACEAE}

Anacamptis collina (Banks et Sol. ex Russel) R.M. Bateman, Pridgeon et M.W. Chase. G bulb. Circum-Medit. WBS.
Anacamptis coriophora (L.) R.M. Bateman, Pridgeon et M.W. Chase. G bulb. Euro-Medit. WBS.

Anacamptis longicornu (Poir.) R.M. Bateman, Pridgeon et M.W. Chase. G bulb. W Medit. WRS.

Anacamptis papilionacea (L.) R.M. Bateman, Pridgeon et M.W. Chase. G bulb. W Medit. WRL.

Anacamptis pyramidalis (L.) Rich. G bulb. Medit.-Atl. NBL.

Gennaria diphylla (Link) Parl. G bulb. Medit.Atl. NBL.

Neotinea lactea (Poir.) R.M. Bateman, Pridgeon et M.W. Chase. G bulb. Circum-Medit. WRS.

Ophrys arachnitiformis Gren. et Philippe. G bulb. Circum-Medit. WRS.

Ophrys bombyliflora Link. G bulb-CircumMedit. WRS.

Ophrys fusca Link subsp. fusca. G bulb. CircumMedit. WRL.

Ophrys incubacea Bianca. G bulb. W Medit. NBS.

Ophrys iricolor Desf. G bulb. Circum-Medit. WRS.

Ophrys lutea Cav. G bulb. Circum-Medit. WRS.

**Ophrys morisii (Martelli) Soò. G bulb. Endem. SA-CO. NBS.

Ophrys neglecta Parl. G bulb. W Medit. NBS.

Ophrys sicula Tineo. G bulb. Circum-Medit. WRS.

Ophrys speculum Link. G bulb. Circum-Medit. WRS.

**Ophrys subfusca (Rchb. f.) Hausskn. subsp. liveranii G. Orrù et M.P. Grasso. G bulb. Endem. SA. NRL.

ophrys x sommieri Camus ex Cortesi [ $O$. bombyliflora x O. neglecta]. G bulb. CircumMedit. WRS.

Orchis anthrophophora (L.) All. G bulb. Medit.Atl. NBS.

Orchis intacta Link. G bulb. Medit.-Atl. NBS.

Serapias lingua L. G bulb. Medit.-Atl. NBL.

Serapias parviflora Parl. G bulb. Medit.-Atl. NBS.

Spiranthes spiralis (L.) Chevall. G rhiz. Medit.Atl. NRS.

XANTHORRHOEACEAE

Asphodelus fistulosus L. H bienn. Circum-Medit. 
WRS.

Asphodelus ramosus L. subsp. ramosus var. ramosus. G rhiz. Circum-Medit. WBL.

\section{DIOSCOREACEAE}

Heteranthera rotundifolia (Kunt) Griseb. I rad. Avv. (Neotrop.). WRL.

Tamus communis L. G rad. Medit.-Atl. NBL.

SMILACACEAE

Smilax aspera L. NP. Circum-Medit. WBL.

\section{ARECACEAE}

Chamaerops humilis L. NP/P scap. W Medit. NBL.

\section{CYPERACEAE}

Bolboschoenus maritimus (L.) Palla - G rhiz. Cosmop. WBL.

Carex distachya Desf. H caesp. Circum-Medit. WRS.

Carex distans L. H caesp. Euro-Medit. WRS.

Carex divisa Huds. G rhiz. Medit.-Atl. WRS.

Carex estensa Gooden. H caesp. Boreo-Trop. WBL.

Carex flacca Schreber subsp. serrulata (Biv.) Greuter. G rhiz. Medit.-Irano-Turan. WRS.

Carex halleriana Asso. H caesp. Circum-Medit. WRL.

Cyperus capitatus Vand. G rhiz. Circum-Medit. WRL.

Cyperus laevigatus L. var. distachyos (All.) Coss. et Durieu. G rhiz. Medit.-Irano-Turan. WRS.

Isolepis cernua (Vahl) Roem. et Schult. T scap. Cosmop. WBL.

Schoenus nigricans L. H caesp. Boreo-Trop. WBL.

Scirpoides holoschoenus (L.) Sojàk. G rhiz Euro-Medit.-Irano-Turan. WRL.

\section{JUNCACEAE}

Juncus acutus L. subsp. acutus. H caesp. EuroMedit. WRL.

Juncus bufonius L. T caesp. Boreo-Trop. WBS.

Juncus hybridus Brot. T caesp. Euro-Medit. WRS.

Juncus maritimus Lam. G rhiz. Euro-Medit. WRS.

Juncus subulatus Forssk. G rhiz. Circum-Medit. WRL.
POACEAE

Aeluropus littoralis (Gouan) Parl. G rhiz. Medit.Irano-Turan. WBS.

Agrostis stolonifera L. H rept. Circumbor. WBL. Aira caryophyllea L. subsp. caryophyllea. T scap. Medit.-Atl. NBL.

Aira cupaniana Guss. T scap. W Medit. NBL.

Aira elegantissima Schur. T scap. Euro-Medit. WRL.

Ammophila arenaria (L.) Link subsp. australis (Mabille) Laínz. G rhiz. Circum-Medit. WRS. Arundo donax L. G rhiz. Circum-Medit. WBL. Arundo collina Ten. G rhiz. Circum-Medit. WRS. Avena barbata Pott ex Link. T scap. Cosmop. WBL.

Avena fatua L. T scap. Cosmop. WBL.

Avena sativa L. T scap. Nat. (Eurasiat.). WBL.

Avena sterilis L. T scap. Circum-Medit. WBL.

*Brachypodium plukenetii P. Beauv. H caesp. Medit.

Brachypodium retusum (Pers.) P. Beauv. H caesp. Circum-Medit. WRL.

Briza maxima L. T scap. Circum-Medit. WBL.

Briza minor L. T scap. Circum-Medit. WBL.

Bromus alopecuros Poir. T scap. Circum-Medit. WBL.

Bromus diandrus Roth subsp. maximus (Desf.) Soó. T scap. Circum-Medit. WBL.

Bromus hordeaceus L. T scap. Paleotemp. WBL.

Bromus hordeaceus L. subsp. thominei (Hardouin) Braun-Blanq. T scap. Euro-Medit. WBS.

Bromus madritensis L. T scap. Medit.-Atl. NBL. Bromus sterilis L. T scap. Paleotemp. WBL.

Bromus tectorum L. T scap. Paleotemp. WBL.

Catapodium balearicum (Willk.) H. Scholz. T scap. Circum-Medit. WBS.

Catapodium rigidum (L.) C.E. Hubb. ex Dony s.l. T scap. Euro-Medit.-Irano-Turan. WBS.

Corynephorus divaricatus (Pourr.) Breistr. T scap. Circum-Medit. WBS.

Crypsis aculeata (L.) Aiton. T scap. Paleotemp. WBL.

Cutandia divaricata (Desf.) Benth. T scap. SW Medit. NBL.

Cutandia maritima (L.) W. Barbey. T scap. Circum-Medit. WRL.

Cynodon dactylon (L.) Pers. H rept. Boreo-Trop. WBL.

Cynosurus cristatus L. H caesp. Paleotemp. WBS. 
Cynosurus echinatus L. T scap. Circum-Medit. WBL.

Cynosurus effusus Link. T scap. Circum-Medit. WBL.

Dactylis glomerata L. subsp. glomerata. H caesp. Paleotemp. WBS.

Dactylis glomerata L. subsp. hispanica (Roth.) Nyman. H caesp. Circum-Medit. WBS.

Dasypyrum villosum (L.) P. Candargy. T scap. Medit.-Irano-Turan. WBS.

Desmazeria sicula (Jacq.) Dumort. T scap. C Medit. NBL.

Echinochloa colona (L.) Link. T scap. BoreoTrop. WBL.

Echinochloa crus-galli (L.) Beauv. T scap. Boreo-Trop. WBS.

Elymus elongatus (Host) Runemark subsp. elongatus. H caesp. Euro-Medit. WBS.

Elymus farctus (Viv.) Runemark ex Melderis subsp. farctus. G rhiz. Circum-Medit. WRS.

Elymus repens (L.) Gould. subsp. repens. G rhiz. Paleotemp. WBL.

Elytrigia repens (L.) Desv. ex Nevski subsp. repens. G rhiz. Paleotemp. WBL.

*Festuca arundinacea Schreb. subsp. fenas (Lag.) Arcang. H caesp. Paleotemp.

Gastridium ventricosum (Gouan) Schinz et Thell. T scap. Circum-Medit. WBS

Gaudinia fragilis (L.) P. Beauv. T scap. CircumMedit. WBS.

Hainardia cylindrica (Willd.) Greuter. T scap. Circum-Medit. WBS.

*Haynaldoticum sardoum Meletti et Onnis. T scap. Circum-Medit.

Hordeum marinum Huds. subsp. marinum. T scap. Circumbor. WBL.

Hordeum murinum L. subsp. leporinum (Link) Arcang. T scap. Circum-Medit. WRL.

Hyparrhenia hirta (L.) Stapf subsp. hirta. H caesp. Medit.-Trop. WRS.

Hyparrhenia sinaica (Delile) Llauradó ex G. López. H caesp. Medit.-Trop. WRS.

Imperata cylindrica ( L.) P. Beauv. G rhiz. Boreo-Trop. WBL.

Lagurus ovatus L. subsp. ovatus. T scap. CircumMedit. WBL.

Lagurus ovatus L. subsp. vestitus (Messeri) H. Scholtz. T scap. W Medit. NBS.

Lamarckia aurea (L.) Moench. T scap. Medit.Irano-Turan. WBL.
Lolium perenne L. - H caesp. Circumbor. WBL. Lolium rigidum Gaudin. T scap. Medit.-IranoTuran. WBL.

Melica ciliata L. subsp. ciliata. H caesp. EuroMedit. WBL.

Melica ciliata L. subsp. magnolii (Gren. et Godr.) Husn. H caesp. W Medit. NBL.

Melica minuta L. H caesp. Circum-Medit. WBL.

Parapholis filiformis (Roth) C.E. Hubb. T scap. W Medit. NBL.

Parapholis incurva (L.) C.E. Hubb. T scap. Medit.-Atl. NBL.

Parapholis marginata Runemark. T scap. S Medit. NBL.

Parapholis strigosa (Dumort.) C.E. Hubb. T scap. Medit.-Atl. NBL.

Paspalum distichum L. G rhiz. Nat. (Neotrop.) WBS.

Paspalum vaginatum Sw. G rhiz. Cosmop. WBL.

Phalaris aquatica L. H caesp. Circum-Medit. WRL.

Phalaris coerulescens Desf. H caesp. CircumMedit. WRL.

Phalaris minor Retz. T scap. Medit-Irano-Turan. WBS.

Phalaris paradoxa L. T scap. Circum-Medit. WBS.

Phragmites australis (Cav.) Trin. ex Steud. G rhiz. Cosmop. WBL.

Piptatherum caerulescens (Desf.) P. Beauv. H caesp. W Medit. NBS.

Piptatherum miliaceum (L.) Coss. subsp. miliaceum. H caesp. Medit.-Atl. WBL.

Poa annua L. T caesp. Cosmop. WBL.

Poa bulbosa L. H caesp. Paleotemp. WBS.

Poa infirma Kunt. T caesp. Euro-Medit. WBS.

Polypogon maritimus Willd. T scap. Medit.Irano-Turan. WRL.

Polypogon monspeliensis (L.) Desf. T scap. Medit.-Trop. WRL.

Polypogon subspathaceus Req. T scap. CircumMedit. WBS.

Puccinellia convoluta (Hornem.) Hayek. H caesp. Circum-Medit. WBS.

Rostraria cristata (L.) Tzvelev. T scap. Medit.Irano-Turan. WRS.

Rostraria hispida (Savi) Dogan. T scap. W Medit. WRS.

Rostraria litorea (All.) Holub. T scap. CircumMedit. WRS. 
Setaria pumila (Poir.) Roem et Schult. T scap. Boreo-Trop. WBL.

Setaria verticillata (L.) P. Beauv. T scap. BoreoTrop. WBL.

Setaria viridis (L.) P. Beauv. T scap. Circumbor. WBS.

Spartina versicolor Fabre. G rhiz. Medit.-Atl. WRL.

Sporobolus virginicus Kunth. G rhiz. CircumMedit. WBL.

Stipa capensis Thunb. T scap. Circum-Medit. WBS.

Trachynia distachya (L.) Link. T scap. Medit.Irano-Turan. WBS.

Triticum ovatum (L.) Raspail. T scap. Medit.Irano-Turan. WBS.

Triticum ventricosum (Tausch) Cesati, Passerini et Gibelli. T scap. W Medit. NBL.

Vulpia bromoides (L.) Gray. T caesp. BoreoTrop. WBL.

Vulpia ciliata Dumort. T caesp. Circum-Medit. WBS.

Vulpia fasciculata (Forssk.) Fritsch. T caesp. Circum-Medit. WBS.

Vulpia geniculata (L.) Link. T caesp. W Medit. NBL.

Vulpia ligustica (All.) Link. T caesp. CircumMedit. WBS.

Vulpia myuros (L.) C.C. Gmel. T caesp. BoreoTrop. WBL.

Vulpia sicula (C. Presl) Link. H caesp. W Medit. NBL.

TYPHACEAE

Typha angustifolia L. subsp. angustifolia. G rhiz. Cosmop. WBS.

Typha latifolia L. G rhiz. Cosmop. WRS.

\section{PAPAVERACEAE}

Fumaria bastardii Boreau. T scap. Medit.-Atl. NBL.

Fumaria bicolor Sommier. T scap. CircumMedit. WRS.

Fumaria capreolata L. T scap. Euro-Medit. WBS.

Fumaria densiflora DC. T scap. Circumbor. WBS.

Fumaria officinalis L. T scap. Paleotemp. WBS. Fumaria parviflora Lam. T scap. Paleotemp.
WBS.

Glaucium corniculatum (L.) Rudolph subsp. corniculatum. T scap. Euro-Medit.-IranoTuran. WRS.

Glaucium flavum Crantz. H scap. Circumbor. WBS.

Hypecoum procumbens L. subsp. procumbens. T scap. Medit.-Irano-Turan. WRS.

Papaver hybridum L. T scap. Paleotemp. WBS.

Papaver rhoeas L. subsp. rhoeas. T scap. Paleotemp. WBL.

Papaver setigerum DC. T scap. Circum-Medit. WBS.

\section{RANUNCULACEAE}

Adonis microcarpa DC. subsp. microcarpa. T scap. Circum-Medit. WBL.

Anemone hortensis L. subsp. hortensis. G bulb. Circum-Medit. WRL.

Clematis cirrhosa L. P lian. Circum-Medit. WBS.

Clematis flammula L. P lian. Euro-Medit. WRS.

**Delphinium longipes Moris. T scap. Endem. SA. NRS.

**Delphinium pictum subsp. pictum Willd. H scap. Endem. SA-CO-BL-H. NRL.

Nigella damascena $\mathrm{L}$. T scap. Circum-Medit. WBL.

Ranunculus aquatilis L. I rad. Boreo-Trop. WBS.

Ranunculus bullatus L. H ros. N Medit. WRS.

**Ranunculus cordiger Viv. subsp. diffusus (Moris) Arrigoni. H scap. Endem. SA-CO. NRS.

Ranunculus muricatus L. T scap. Circum-Medit. WBS.

Ranunculus parviflorus L. T scap. Medit.-Atl. NBL.

Ranunculus peltatus Schrank. I rad. Paleotemp. WBS.

Ranunculus trilobus Desf. T scap. W Medit. NBL.

\section{AIZOACEAE}

Carpobrotus acinaciformis (L.) L. Bolus. Ch frut. Nat. (Sudafr.). WBL.

Carpobrotus edulis (L.) N.E. Br. Ch frut. Nat. (Sudafr.). WBL.

Mesembryanthemum nodiflorum L. T scap. Medit.-Trop. WBS. 


\section{AMARANTHACEAE \\ Amaranthus cruentus L. T scap. Avv. (Neotrop.). WBS.}

Arthrocnemum macrostachyum (Moric.) Moris. Ch succ. Medit.-Irano-Turan. WRL.

Atriplex halimus L. P caesp. Circum-Medit. WBL.

Atriplex patula L. T scap. Circumbor. WBS.

Atriplex portulacoides L. Ch frut. Circumbor. WBL.

Atriplex prostrata Boucher ex DC. T scap. Circumbor. WBS.

Atriplex rosea L. T scap. Euro-Medit. WRS.

Bassia hirsuta (L.) Asch. T scap. Eurasiat. WBS.

Bassia scoparia (L.) A.J. Scott subsp. scoparia. T scap. Avv. (Eurasiat.). WBL.

Beta vulgaris L. subsp. maritima (L.) Arcang. T scap. Euro-Medit. WBS.

Camphorosma monspeliaca L. Ch frut. Medit.Irano-Turan. WBS.

Chenopodium album L. subsp. album. T scap. Cosmop. WBL.

Chenopodium ambrosioides $\mathrm{L}$. T scap. Nat. (Neotrop.). WBL.

Chenopodium murale L. T scap. Boreo-Trop. WBS.

Chenopodium urbicum L. T scap. Circumbor. WBS.

Chenopodium vulvaria L. T scap. Euro-Medit. WRL.

Salicornia patula Duval-Jouve. T scap. CircumMedit. WRL.

Salsola kali L. subsp. kali. T scap. Circumbor. WBL.

Salsola soda L. T scap. Paleotemp. WBS.

Sarcocornia fruticosa (L.) A.J. Scott. Ch succ. Boreo-Trop. WRS.

Sarcocornia perennis (Mill.) A.J. Scott. Ch succ. Euro-Medit. WRL.

Suaeda maritima (L.) Dumort. T scap. Cosmop. WRL.

Suaeda vera J.F. Gmel. NP caesp. Medit.-Atl. WRL.

\section{CACTACEAE}

Opuntia amyclaea Mill. P succ. Nat. (Neotrop.). WBS.

CARYOPHYLLACEAE

Arenaria serpyllifolia L. subsp. serpyllifolia. T scap. Boreo-Trop. WBL.

Cerastium glomeratum Thuill. T scap. Circumbor. WBS.

*Cerastium pumilum Curtis. T scap. Euro-Medit.

Cerastium semidecandrum L. T scap. Cosmop. WBS.

Minuartia hybrida (Vill.) Schischk. subsp. hybrida. T scap. Paleotemp. WBL.

Minuartia mediterranea (Link) K. Maly. T scap. Circum-Medit. WBS.

Paronychia argentea Lam. H caesp. CircumMedit. WBS.

Paronychia echinulata Chater. T scap. CircumMedit. WBS.

Petrorhagia prolifera (L.) P.W. Ball et Heywood. T scap. Euro-Medit. WBS.

Polycarpon tetraphyllum (L.) L. subsp. alsinifolium (Biv.) Ball. T scap. Medit.-IranoTuran. WRS.

Polycarpon tetraphyllum (L.) L. subsp. diphyllum (Cav.) O. Bolòs et Font Quer. T scap. Circum-Medit. WRS.

Rhodalsine geniculata (Poir.) F.N. Williams. Ch suffr. W Medit. WRS.

Sagina apetala Ard. subsp. apetala. T scap. Paleotemp. WBS.

Sagina maritima G. Don. T scap. Medit.-Atl. WBS.

Silene arghireica Vals. T scap. W Medit. NRL.

**Silene beguinotii Vals. T scap. Endem. SA. NRS.

Silene canescens Ten. T scap. Circum-Medit. WBS.

Silene coelirosa (L.) Godr. T scap. W Medit. WBS.

Silene colorata Poir. T scap. Circum-Medit. WBS.

Silene cucubalus Wibel. H scap. Subcosmop. WBL.

Silene gallica L. T scap. Euro-Medit. WBS.

Silene latifolia Poir. H bienn. Circum-Medit. WBS.

Silene niceensis All. T scap. Circum-Medit. WBS.

Silene nocturna L. T scap. Circum-Medit. WBS.

Silene nummica Vals. T caesp. C Medit. NBS.

**Silene succulenta Forssk. subsp. corsica (DC.) Nyman. H scap. Endem. SA-CO. NRS.

Silene vulgaris (Moench) Garcke subsp. tenoreana (Colla) Soldano et $\mathrm{F}$. Conti. H scap. 
Circum-Medit. WBL.

*Silene vulgaris (Moench) Garcke subsp. vulgaris. T scap. Circumbor.

Spergularia rubra (L.) J. et C. Presl. H caesp. Subcosmop. WBS.

Spergularia salina J. et C. Presl. T scap. Subcosmop. WBS.

Stellaria media (L.) Vill. subsp. media. T rept. Cosmop. WBS.

Stellaria pallida (Dumort.) Pirè. T scap. EuroMedit. WBS.

Vaccaria hispanica (Mill.) Rauschert. T scap. Paleotemp. WBS.

\section{FRANKENIACEAE}

Frankenia hirsuta L. Ch suffr. Medit.-IranoTuran. WRS.

Frankenia laevis L. subsp. laevis. Ch suffr. Subcosmop. WBS.

Frankenia pulverulenta $\mathrm{L}$. subsp. pulverulenta. T scap. Medit.-Irano-Turan. WRS.

\section{PORTULACACEAE}

Portulaca oleracea L. subsp. oleracea. T scap. Boreo-Trop. WBS.

\section{PLUMBAGINACEAE}

Armeria pungens (Link) Hoffmanns. et Link. Ch suffr. W Medit. NRS.

**Limonium acutifolium (Rchb.) Salmon subsp. acutifolium. Ch suffr. Endem. SA-CO. NRS.

**Limonium capitis-marci Arrigoni et Diana. Ch suffr. Endem. SA. NRS

**Limonium cornusianum Arrigoni et Diana. Ch suffr. Endem. SA. NRS.

Limonium divaricatum (Rouy) Brullo. Ch suffr. W Medit. NRL.

** Limonium dubium (Andr. ex Guss.) Litard. H ros. Endem. SA-CO-SI. NRL.

**Limonium glomeratum (Tausch) Erben. Ch suffr. Endem. SA-SI. NRL.

**Limonium lausianum Pignatti. Ch suffr. Endem. SA. NRS.

Limonium narbonense Mill. H ros. CircumMedit. WBS.

**Limonium oristanum Mayer. Ch suffr. Endem. SA. NRS.

**Limonium pseudolaetum Arrigoni et Diana. Ch suffr. Endem. SA. NRS.

**Limonium tenuifolium (Bertol. ex Moris)
Erben. Ch suffr. Endem. SA. NRS.

**Limonium tharrosianum Arrigoni et Diana. Ch suffr. Endem. SA. NRS.

Limonium virgatum (Willd.) Fourr. H ros. EuroMedit. WRL.

Plumbago europaea L. Ch frut. Euro-Medit. WBS.

\section{POLYGONACEAE}

Emex spinosa (L.) Campd. T scap. CircumMedit. WBS.

Fallopia convolvulus (L.) Á Löve. T scap. Circumbor. WBS.

Polygonum aviculare L. T rept. Boreo-Trop. WBS.

Polygonum maritimum L. Ch rept. Medit.-Atl. WBS.

*Polygonum patulum Bieb. T scap. Euro-Medit.

**Polygonum scoparium Req. ex Loisel. Ch frut. Endem. SA-CO. NRS.

Rumex bucephalophorus L. s.l. T scap. CircumMedit. WBL.

Rumex conglomeratus Murray. H scap. Circumbor. WBL.

Rumex crispus L. H scap. Paleotemp. WBL.

Rumex obtusifolius L. H scap. Boreo-Trop. WBS.

Rumex pulcher L. H scap. Medit.-Atl. WBS.

\section{TAMARICACEAE}

Tamarix africana Poir. P caesp. W Medit. NBS.

*Tamarix arborea Ehremb. ex Bunge. P caesp. SE Medit.

*Tamarix canariensis Willd. P caesp. SW Medit.

*Tamarix dalmatica Baum. P scap. E Medit.

Tamarix gallica L. P caesp. W Medit. NBS.

*Tamarix tetragyna Ehremb. NP. Medit.-IranoTuran.

\section{SANTALACEAE}

Osyris alba L. NP. Circum-Medit. WRL.

Thesium humile Vahl. T scap. Circum-Medit. WRS.

\section{CRASSULACEAE}

Sedum caeruleum L. T scap. S Medit. WRS. Sedum litoreum Guss. T scap. Euro-Medit. WRS. Sedum rubens L. T scap. Medit.-Atl. WRS.

Sedum sediforme (Jacq.) Pau. Ch succ. CircumMedit. WRL. 
Sedum stellatum L. T scap. Circum-Medit. WRL. Tillaea muscosa L. T scap. Euro-Medit. WRS.

Tillaea vaillantii Willd. T scap. Boreo-Trop. WRS.

Umbilicus gaditanus Boiss. G bulb. Medit.-Trop. WRS.

Umbilicus rupestris (Salisb.) Dandy. G bulb. Medit.-Trop. WRS.

\section{HALORAGACEAE}

Myriophyllum spicatum L. I rad. Boreo-Trop. WBS.

\section{GERANIACEAE}

Erodium cicutarium (L.) L'Hér. T scap. Paleotemp. WBS.

Erodium laciniatum (Cav.) Willd. T scap. Circum-Medit. WBS.

Erodium moschatum (L.) L'Hér. T scap. CircumMedit. WBS.

Geranium molle L. T scap. Paleotemp. WBS.

Geranium purpureum Vill. T scap. Paleotemp. WRL.

Geranium robertianum L. T scap. Circumbor. WBL.

\section{LYTHRACEAE}

Lythrum hyssopifolia L. T scap. Paleotemp. WBS.

Lythrum junceum Banks et Sol. H scap. CircumMedit. WBS.

Middendorfia borystenica (Schrank) Trautv. T scap. Euro-Medit. WBS.

\section{MYRTACEAE}

Eucalyptus camaldulensis Dehnh. P scap. Nat. (Australia). WBL.

Myrtus communis L. subsp. communis. P caesp. Circum-Medit. WRL.

\section{FABACEAE}

Acacia saligna (Labill.) H.L. Wendl. P caesp. Nat. (Australia). WBL.

Anagyris foetida L. P caesp. Circum-Medit. WBS.

Astragalus boeticus L. T scap. Medit.-IranoTuran. WBS.

Astragalus hamosus L. T scap. Circum-Medit. WBS.

**Astragalus pelecinus (L.) Barneby subsp. pelecinus. T scap. Endem. SA-CO-AT-SI-ITM. NBS.

Bituminaria bituminosa (L.) C.H. Stirt. H scap. Circum-Medit. WRS.

Calicotome villosa (Poir.) Link. P caesp. CircumMedit. WRL.

Coronilla scorpioides (L.) W.D.J. Koch. T scap. Medit.-Irano-Turan. WBS.

Cytisus villosus Pourr. P caesp. Circum-Medit. WRS.

Dorycnium herbaceum Vill. H scap. Euro-Medit. WRL.

Dorycnium hirsutum (L.) Ser. Ch suffr. CircumMedit. WRL.

**Genista corsica (Loisel.) DC. NP. Endem. SACO. NBL.

*Hippocrepis biflora Spreng. T scap. CircumMedit.

Hippocrepis ciliata Willd. T scap. Euro-Medit. WBS.

Hippocrepis multisiliquosa L. T scap. W Medit. WRS.

Lathyrus aphaca L. T scap. Euro-Medit. WBL.

Lathyrus cicera L. T scap. Circum-Medit. WBS.

Lathyrus clymenum L. T scap. Circum-Medit. WBS.

Lathyrus ochrus (L.) DC. T scap. Circum-Medit. WBS.

Lotus angustissimus L. T scap. Euro-Medit.Irano-Turan. WBS.

Lotus conimbricensis Brot. T scap. CircumMedit. WRL.

Lotus corniculatus L. H scap. Paleotemp. WBL.

**Lotus cytisoides L. subsp. conradiae Gamisans. Ch suffr. Endem. SA-CO. NRL.

Lotus edulis L. T scap. Circum-Medit. WRL.

Lotus ornithopodioides L. T scap. Circum-Medit. WBS.

Lotus parviflorus Desf. T scap. Medit.-Atl. WRS.

Lupinus gussoneanus Agardh. T scap. CircumMedit. WBS.

Medicago doliata Carmign. T scap. CircumMedit. WBS

**Medicago intertexta (L.) Mill. var. tuberculata Moris. T scap. Endem. SA. NRS.

Medicago italica (Mill.) Fiori subsp. tornata (L.) Emb. et Maire. T scap. W Medit. NBS.

Medicago littoralis Loisel. T scap. CircumMedit. WBS.

Medicago marina L. Ch rept. Medit.-Atl. WRS. 
Medicago minima (L.) L. T scap. Euro-Medit. WBS.

Medicago murex Willd. T scap. Circum-Medit. WBS.

Medicago orbicularis (L.) Bartal. T scap. Medit.Irano-Turan. WBS.

Medicago polymorpha L. T scap. Medit.-IranoTuran. WBS.

Medicago praecox DC. T scap. N Medit. WRL.

Medicago rigidula (L.) All. T scap. CircumMedit. WBS.

Medicago sativa L. H scap. Eurasiat. WBL.

Medicago tuberculata (Retz.) Willd. T scap. Circum-Medit. WBS.

Melilotus albus Medik. T scap. Euro-Medit.Irano-Turan. WBS.

Melilotus officinalis (L.) Pallas. H bienn. Subcosmop. WBL.

Melilotus siculus (Turra) Steud. T scap. CircumMedit. WBS.

Melilotus sulcatus Desf. T scap. Circum-Medit. WBS.

Ononis minutissima L. Ch suffr. W Medit. WRS.

Ononis natrix L. subsp. ramosissima (Desf.) Batt. H caesp. Circum-Medit. WBS.

Ononis reclinata L. T scap. Circum-Medit. WBS.

Ononis variegata $\mathrm{L}$. T scap. Circum-Medit. WBS.

Ononis viscosa L. subsp. breviflora (DC.) Nyman. T scap. Circum-Medit. WBS.

Ornithopus compressus L. T scap. CircumMedit. WBL.

Pisum sativum L. subsp. biflorum (Raf.) Soldano. T scap. Circum-Medit. WBS.

Scorpiurus muricatus L. T scap. Circum-Medit. WBL.

Sulla capitata (Desf.) B.H. Choi et $\mathrm{H}$. Ohashi. T scap. Circum-Medit. WBL.

Sulla coronaria (L.) Medik. H scap. W Medit. WBS.

Sulla spinosissima (L.) B.H. Choi et H. Ohashi. T scap. W Medit. WBS.

Trifolium angustifolium L. T scap. CircumMedit. WBL.

Trifolium arvense L. T scap. Euro-Medit.-IranoTuran. WBL.

Trifolium bocconei Savi. T scap. Circum-Medit. WBS.

Trifolium campestre Schreb. T scap. Euro-Medit. WBL.

Trifolium cherleri L. T scap. Circum-Medit.
WRS.

Trifolium glomeratum L. T scap. Medit.-Atl. WRL.

Trifolium incarnatum L. subsp. molinerii (Hornem.) Ces. T scap. Euro-Medit. WBS.

Trifolium lappaceum L. T scap. Circum-Medit. WBS.

Trifolium nigrescens Viv. T scap. Circum-Medit. WBS.

Trifolium pratense L. T scap. Paleotemp. WBL. Trifolium repens L. H rept. Circumbor. WBL.

Trifolium resupinatum $\mathrm{L}$. T rept. Circum-Medit. WBS.

Trifolium scabrum L. subsp. scabrum. T scap. Circum-Medit. WBL.

Trifolium spumosum L. T scap. Circum-Medit. WBS.

Trifolium squamosum L. T scap. Medit.-Atl. WRL.

Trifolium squarrosum L. T scap. Circum-Medit. WBS.

Trifolium stellatum L. T scap. Medit.-IranoTuran. WBL.

Trifolium subterraneum L. T rept. Euro-Medit. WBS.

Trifolium tomentosum $\mathrm{L}$. T rept. Circum-Medit. WBS.

Tripodion tetraphyllum (L.) Fourr. T scap. Circum-Medit. WRS.

Vicia benghalensis L. T scap. Circum-Medit. WBS.

Vicia lathyroides L. T scap. Euro-Medit. WBS.

Vicia lutea L. T scap. Circum-Medit. WBL.

Vicia parviflora Cav. T scap. Circum-Medit. WBS.

Vicia pubescens (DC.) Link. T scap. CircumMedit. WBS.

*Vicia sativa L. subsp. macrocarpa (Moris) Arcang. T scap. Circum-Medit.

Vicia sativa L. subsp. nigra (L.) Ehrh. T scap. Medit.-Irano-Turan. WBL.

Vicia sativa L. subsp. sativa. T scap. Avv. (Medit.-Turan.). WBL.

Vicia villosa Roth. T scap. Euro-Medit. WBL.

Vicia villosa Roth subsp. varia (Host) Corb. T scap. Euro-Medit. WBS.

\section{POLYGALACEAE}

Polygala monspeliaca L. T scap. Circum-Medit. WBS. 
**Polygala sinisica Arrigoni. Ch suffr. Endem. SA. NRS

\section{FAGACEAE}

Quercus calliprinos Webb. P scap. E Medit. WRS.

Quercus ilex L. P scap. Circum-Medit. WRS.

CLUSIACEAE

Hypericum perfoliatum L. H scap. CircumMedit. WBS.

Hypericum perforatum L. H scap. Paleotemp. WBS.

\section{ELATINACEAE}

Elatine macropoda Guss. I rad. W Medit. WRS.

\section{EUPHORBIACEAE}

Chamaesyce peplis (L.) Prokh. T rept. EuroMedit. WBS.

Chrozophora tinctoria (L.) A. Juss. T scap. Medit.-Irano-Turan. WRS.

Euphorbia characias L. NP. W Medit. WBS.

Euphorbia dendroides L. NP. Circum-Medit. WRS.

Euphorbia exigua L. subsp. exigua. T scap. Euro-Medit. WBS.

Euphorbia helioscopia L. subsp. helioscopia. T scap. Paleotemp. WBS.

Euphorbia lathyris L. H bienn. Nat. (Medit.Turan.). WBS.

Euphorbia paralias L. Ch frut. Medit.-Atl. WRL.

Euphorbia peplus L. T scap. Circumbor. WBL.

**Euphorbia pithyusa L. subsp. cupanii (Guss. ex Bertol.) Radel-Sm. Ch suffr. Endem. SACO-SI. NRL.

**Euphorbia pithyusa L. subsp. pithyusa. Ch suffr. Endem. SA-CO-AT-BL. NBS.

Euphorbia pterococca Brot. T scap. W Medit. WBS.

Euphorbia terracina L. T scap. Circum-Medit. WBS.

Mercurialis annua L. T scap. Paleotemp. WBS.

**Mercurialis corsica Coss. et Kralil. Ch suffr. Endem. SA-CO. NRS.

\section{LINACEAE}

Linum bienne Mill. H bienn. Medit.-Atl. WBS.

Linum strictum L. subsp. strictum. T scap. Medit.-Irano-Turan. WBS.
Linum trigynum L. T scap. Circum-Medit. WBS.

\section{VIOLACEAE}

Viola arborescens L. Ch frut. W Medit. NBL.

OXALIDACEAE

Oxalis pes-caprae L. G bulb. Avv. (S Africa). WBL.

\section{MORACEAE}

Ficus carica L. var. caprificus Risso. P scap. N Medit. WBS.

\section{RHAMNACEAE}

Rhamnus alaternus L. subsp. alaternus. P caesp. Circum-Medit. WBS.

\section{ROSACEAE}

Potentilla reptans L. H ros. Paleotemp. WRS.

Rubus gr. ulmifolius Schott. NP. Euro-Medit. WBL.

Sanguisorba rupicola (Boiss. et Reut.) A. Braun et C.D. Bouché. H scap. W Medit. NBL.

\section{URTICACEAE}

Parietaria judaica L. H scap. Euro-Medit.-IranoTuran. WBS.

Parietaria lusitanica L. subsp. lusitanica. T rept. Circum-Medit. WBS.

**Urtica atrovirens Req. ex Loisel. H scap. Endem. SA-CO-AT. NBS.

Urtica membranacea Poir. ex Savigny. T scap. Circum-Medit. WBS.

Urtica urens L. T scap. Boreo-Trop. WBS.

\section{BRASSICACEAE}

Brassica nigra (L.) W.D.J. Koch. T scap. EuroMedit. WBS.

Brassica rapa L. T scap. Nat. (Medit.). WBS.

Brassica tournefortii Gouan. T scap. Medit.Irano-Turan. WBS.

Bunias erucago L. T scap. Euro-Medit. WBS.

Cakile maritima Scop. subsp. maritima. T scap. Circum-Medit. WRL.

Capsella bursa-pastoris (L.) Medik. subsp. bursa-pastoris. H bienn. Cosmop. WBS.

Capsella rubella Reut. T scap. Circum-Medit. WBS.

Cardamine hirsuta L. T scap. Circumbor. WBS. Diplotaxis viminea (L) DC. T scap. Circum- 
Medit. WBS.

Erophila praecox (Steven) DC. T scap. Paleotemp. WBS.

Erophila verna (L.) Chevall. T scap. Paleotemp. WBS.

Eruca vesicaria (L.) Cav. T scap. Medit.-Turan. WBL.

Hirschfeldia incana (L.) Lagr.-Foss. H scap. Circum-Medit. WBS.

Lepidium graminifolium L. subsp. graminifolium. H scap. Euro-Medit. WBS.

Lepidium graminifolium L. subsp. suffruticosum (L.) P. Monts. Ch suffr. W Medit. WBS.

Lobularia maritima (L.) Desv. subsp. maritima. H scap. Circum-Medit. WBS.

Malcolmia ramosissima (Desf.) Gennari. T scap. W Medit. WRL.

Matthiola incana (L.) R. Br. Ch suffr. W Medit. WRS.

Matthiola sinuata (L.) R. Br. H scap. Medit.-Atl. WRS.

Matthiola tricuspidata (L.) R. Br. T scap. Circum-Medit. WRL.

Raphanus raphanistrum L. subsp. raphanistrum. T scap. Euro-Medit. WBS.

Rapistrum rugosum (L.) Arcang. T scap. EuroMedit. WBS.

\section{RESEDACEAE}

Reseda alba L. H scap. Circum-Medit. WBS. Reseda lutea L. H scap. Euro-Medit. WBS.

Reseda luteola L. H scap. Euro-Medit. WBS.

\section{CISTACEAE}

Cistus creticus L. subsp. eriocephalus (Viv.) Greuter et Burdet. NP. Circum-Medit. WRL.

Cistus monspeliensis L. NP. Circum-Medit. WBL.

Cistus salviifolius L. NP. W Medit. WBL.

Fumana laevipes (L.) Spach. Ch suffr. CircumMedit. WRL.

Fumana thymifolia (L.) Spach ex Webb. Ch suffr. Circum-Medit. WRL.

Helianthemum caput-felis Boiss. Ch suffr. SW Medit. NRL.

Helianthemum salicifolium (L.) Mill. T scap. Euro-Medit. WRS.

Tuberaria guttata (L.) Fourr. T scap. Euro-Medit. WBS.

\section{MALVACEAE}

Alcea rosea L. H scap. Nat. (ignota). WBS. Althaea hirsuta L. T scap. Euro-Medit. WBS.

Lavatera arborea L. H bienn. Circum-Medit. WBS.

Lavatera cretica L. T scap. Circum-Medit. WBS. Lavatera olbia L. P caesp. W Medit. WRS.

Lavatera trimestris L. T scap. Circum-Medit. WBS.

Malva neglecta Wallr. T scap. Euro-Medit.-IranoTuran. WBS.

Malva nicaeensis All. T scap. Circum-Medit. WBS.

Malva parviflora L. T scap. Circum-Medit. WBS.

Malva sylvestris L. subsp. sylvestris. H scap. Euro-Medit. WBL.

\section{THYMELAEACEAE}

Daphne gnidium L. P caesp. Circum-Medit. WRS.

Thymelaea hirsuta (L.) Endl. NP. Circum-Medit. WRL.

Thymelaea tartonraira (L.) All. subsp. tartonraira. NP. Circum-Medit. WRL.

\section{ANACARDIACEAE}

Pistacia lentiscus L. P caesp. Circum-Medit. WBL.

\section{RUTACEAE}

Ruta chalepensis L. Ch suffr. Circum-Medit. WRL.

\section{ERICACEAE}

Arbutus unedo L. P caesp. Medit.-Atl. WRS. Erica multiflora L. NP. W Medit. NBL.

Erica scoparia L. P caesp. Circum-Medit. WRL.

\section{PRIMULACEAE}

Anagallis arvensis L. subsp. latifolia (L.) Arcang. T rept. Boreo-Trop. WBS.

Anagallis arvensis L. subsp. parviflora (Hoffmanns. et Link) Arcang. T rept. W Medit. WBS.

Anagallis foemina Mill. T rept. Boreo-Trop. WBS.

Asterolinon linum-stellatum (L.) Duby. T scap. Circum-Medit. WRS.

Coris monspeliensis L. subsp. monspeliensis. H bienn. W Medit. NRL. 


\section{BORAGINACEAE}

Anchusa azurea Mill. H scap. Euro-Medit.-IranoTuran. WRS.

***Anchusa littorea Moris. T scap. Endem. SA

Borago officinalis L. T scap. Circum-Medit. WBS Cerinthe major L. T scap. Circum-Medit. WBL.

Cynoglossum cheirifolium L. subsp. cheirifolium. $\mathrm{H}$ bienn. W Medit. WBS.

Cynoglossum creticum Mill. H bienn. Medit.-IranoTuran. WBS.

Cynoglossum officinale L. H bienn. Euro-Medit. WBS.

Echium arenarium Guss. H bienn. Circum-Medit WRS.

Echium creticum L. H bienn. W Medit. WBS.

Echium italicum L. H bienn. Euro-Medit. WBS.

Echium plantagineum L. H bienn. Circum-Medit. WBL.

Echium sabulicola Pomel subsp. sabulicola. H scap. W Medit. WRS.

Heliotropium europaeum L. T scap. Euro-Medit.Irano-Turan. WBS.

Myosotis arvensis (L.) Hill subsp. arvensis. T scap. Euro-Medit. WRS.

Neatostema apulum (L.) I.M. Johnst. T scap. Circum-Medit. WBS.

\section{APOCYNACEAE}

Nerium oleander L. subsp. oleander. P caesp. Circum-Medit. WBS.

\section{GENTIANACEAE}

Blackstonia grandiflora (Viv.) Pau. T scap. W Medit. WBS.

Blackstonia perfoliata (L.) Huds. T scap. EuroMedit. WBS.

Centaurium erythraea Rafn. subsp. majus (Hoffmanns. et Link) Melderis. H bienn. W Medit. WRL.

**Centaurium erythraea Rafn. subsp. rhodense (Boiss. et Reut.) Melderis. T scap. Endem. SACO-SI-ITM. NRL.

Centaurium maritimum (L.) Fritsch. T scap. Circum-Medit. WRL.

Centaurium pulchellum (Sw.) Druce subsp. pulchellum. T scap. Circumbor. WRL.

Centaurium spicatum (L.) Fritsch. T scap. CircumMedit. WBS.

Centaurium tenuiflorum (Hoffmanns. et Link) Fritsch subsp. acutiflorum (Schott) Zeltner. T scap. Circum-Medit. WBS.

Cicendia filiformis (L.) Delarbre. T scap. EuroMedit. WBL.

\section{RUBIACEAE}

Asperula arvensis L. T scap. Euro-Medit. WBS.

Crucianella maritima L. Ch suffr. Circum-Medit. WRL.

Galium aparine L. T scap. Paleotemp. WBS.

Galium murale (L.) All. T scap. Circum-Medit. WBS.

Galium palustre L. H scap. Circumbor. WRS.

Galium spurium L. T scap. Euro-Medit.-IranoTuran. WBS.

Galium verrucosum Huds. T scap. Euro-Medit. WBS.

***Galium verrucosum Huds. var. halophilum (Ponzo) Natali et Jeanmonod. T scap. Endem. SA-CO-AT-SI.

Rubia peregrina $\mathrm{L}$. subsp. peregrina. $\mathrm{P}$ lian. Circum-Medit. WBL.

**Rubia peregrina L. subsp. requienii (Duby) Cardona et Sierra-Ràfols. P lian. Endem. SACO-ITM. NBS.

Sherardia arvensis L. T scap. Euro-Medit. WBL. Theligonum cynocrambe L. T scap. CircumMedit. WBS.

Valantia hispida L. T scap. S Medit. WBS.

Valantia muralis L. T scap. Circum-Medit. WBS.

\section{LAMIACEAE}

Ajuga iva (L.) Schreb. subsp. iva. Ch suffr. Circum-Medit. WBS.

Ajuga iva (L.) Schreb. subsp. pseudoiva (DC.) Briq. Ch suffr. SW Medit. WBS.

Lamium amplexicaule L. T scap. Paleotemp. WBS.

Lavandula stoechas L. subsp. stoechas. NP. Circum-Medit. WRS.

Lycopus europaeus L. H scap. Paleotemp. WBS. Marrubium vulgare L. H scap. Paleotemp. WBS. Mentha pulegium L. subsp. pulegium. H scap. Euro-Medit.-Irano-Turan. WBS

**Micromeria filiformis (Aiton) Benth. subsp. filiformis. Ch suffr. Endem. SA-BL. NRS.

Micromeria graeca (L.) Benth. ex Rchb. subsp. graeca. Ch suffr. Circum-Medit. WBS.

Prasium majus L. Ch frut. Circum-Medit. WBS.

Rosmarinus officinalis L. NP. Circum-Medit. WBL. 
**Rosmarinus officinalis L. var. palaui $\mathrm{O}$. Bolos et R. Mol. NP. Endem. SA-CO-BL. NRL.

Salvia verbenaca $\mathrm{L}$. H scap. Medit.-Atl. WBS.

Sideritis romana $\mathrm{L}$. subsp. romana. T scap. W Medit. WBS.

Stachis arvensis (L.) L. T scap. Euro-Medit. WBS.

**Stachys glutinosa L. Ch frut. Endem. SA-COAT. NBS.

Stachys ocymastrum (L.) Briq. T scap. W Medit. WBS.

Teucrium capitatum L. subsp. capitatum. Ch suffr. Circum-Medit. WBS.

*Teucrium fruticans L. NP. W Medit.

**Teucrium marum L. subsp. marum. Ch frut. Endem. SA-CO-AT-BL-H. NRL.

**Teucrium subspinosum Pourr. ex Willd. Ch frut. Endem. SA-BL. NRS.

\section{OLEACEAE}

Olea europea L. var. sylvestris Brot. $\mathrm{P}$ caesp/P scap. Circum-Medit. WBL.

Phillyrea angustifolia L. P caesp. Circum-Medit. WBL.

Phillyrea latifolia L. P scap. Circum-Medit. WBL.

**Phillyrea media L. var. rodriguezii $\mathrm{P}$. Monts. P caesp. Endem SA-CO-BL. NRL.

\section{OROBANCHACEAE}

Bartsia trixago L. T scap. Circum-Medit. WBS.

*Orobanche amethystea Thuill. subsp. amethystea. T par. Euro-Medit.

Orobanche minor Sm. T par. Euro-Medit-IranoTuran. WRS.

Orobanche sanguinea C. Presl. T par. W Medit. WRS.

Phelipanche mutelii (F.W. Schultz) Reut. T par. Circum-Medit. WRS.

Parentucellia viscosa (L.) Caruel. T scap. Medit.Atl. WBS.

\section{PLANTAGINACEAE}

Kickxia commutata (Bernh. ex Rchb.) Fritsch. $\mathrm{H}$ rept. Circum-Medit. WBS.

Kickxia elatine (L.) Dumort. T scap. Euro-Medit. WBS.

Kickxia spuria (L.) Dumort. T scap. Euro-Medit. WBS.

Linaria pelisseriana (L.) Mill. T scap. Medit.-
Atl. WBS.

Linaria reflexa (L.) Desf. T rept. C Medit. WBS.

Linaria triphylla (L.) Mill. T scap. W Medit. WRS.

Misopates orontium (L.) Raf. subsp. orontium. T scap. Paleotemp. WBS.

Plantago bellardii All. T scap. Circum-Medit. WBL.

Plantago coronopus L. subsp. coronopus. T scap. Circum-Medit. WBL.

Plantago crassifolia Forssk. H ros. CircumMedit. WRL.

Plantago lagopus L. T scap. Circum-Medit. WBL.

Plantago lanceolata L. H ros. Circumbor. WBS.

Plantago macrorrhiza Poir. H ros. W Medit. WBS.

Plantago major L. H ros. Cosmop. WBS.

Plantago maritima L. H ros. Circumbor. WRL.

Plantago weldenii Rchb. T scap. Paleotemp. WBS.

\section{SCROPHULARIACEAE}

**Scrophularia ramosissima Loisel. Ch suffr. Endem. SA-CO-BL-GA. NRL.

**Scrophularia trifoliata L. H scap. Endem. SACO-AT. NBS.

Verbascum pulverulentum Vill. H bienn. EuroMedit. WBS.

Verbascum sinuatum L. H bienn. Circum-Medit. WBS.

\section{VERBENACEAE}

Verbena officinalis L. H scap. Boreo-Trop. WBS.

\section{CONVOLVULACEAE}

Calystegia soldanella (L.) Roem et Schult. G rhiz. Cosmop. WRL.

Convolvulus althaeoides L. H scand. CircumMedit. WBL.

Convolvulus arvensis $\mathrm{L} . \mathrm{G}$ rhiz. Paleotemp. WBS.

Cressa cretica L. Ch suffr. Cosmop. WRL.

Cuscuta epithymum (L.) L. T par. Euro-Medit. WRS.

Cuscuta planiflora Ten. T par. Circum-Medit. WRS.

\section{SOLANACEAE}

Datura stramonium L. subsp. stramonium. T 
scap. Boreo-Trop. WBS.

Hyoscyamus albus L. H bienn. Circum-Medit. WBS.

Solanum nigrum L. subsp. nigrum. T scap. Boreo-Trop. WBS.

Solanum sodomaeum L. NP. Nat. (Africa). WBS.

\section{APIACEAE}

Ammi majus L. T scap. Circum-Medit. WBS. Ammoides pusilla (Brot.) Breistr. T scap. Circum-Medit. WRS.

Apium graveolens L. H scap. Paleotemp. WRS. Apium nodiflorum (L.) Lag. I rad. Paleotemp. WRS.

Bupleurum baldense Turra. T scap. Euro-Medit. WBS.

*Bupleurum lancifolium Hornem. T scap. Medit.-Irano-Turan.

Bupleurum semicompositum L. T scap. Medit. Irano-Turan. WBS.

Crithmum maritimum L. Ch suffr. Medit.-Atl. WRL.

Daucus carota L. subsp. carota. H scap. EuroMedit. WBL.

Daucus carota L. subsp. drepanensis (Arcang.) Heywood. H bienn. Circum-Medit. WBS.

Daucus carota L. subsp. maritimus (Lam.) Batt. H bienn. W Medit. WBL.

Daucus carota L. subsp. maximus (Desf.) Ball. H bienn. Circum-Medit. WBS.

Daucus pumilus (L.) Hoffmanns. et Link. T scap. Circum-Medit. WBS.

Echinophora spinosa L. H scap. Medit.-Atl. WRS.

Eryngium barrelieri Boiss. H bienn. CircumMedit. WRS.

Eryngium campestre L. H scap. Euro-Medit.Irano-Turan. WBS.

Eryngium maritimum L. G rhiz. Medit.-Atl. WRL.

Eryngium tricuspidatum L. H scap. SW Medit. WBS.

**Ferula arrigonii Bocchieri. H scap. Endem. SA-CO. NRS.

Ferula communis L. H scap. Circum-Medit. WRL.

Foeniculum vulgare Mill. subsp. piperitum (Ucria) Cout. H scap. S Medit. WBS.

Hydrocotyle ranunculoides $\mathrm{L}$. $f$. G rhiz. BoreoTrop. WRL.
Scandix pecten-veneris L. T scap. Euro-Medit. WRS.

Smyrnium olusatrum L. H bienn. Circum-Medit. WBS.

Thapsia garganica L. H scap. Circum-Medit. WBS.

Torilis arvensis (Huds.) Link subsp. purpurea (Ten.) Hayek. T scap. Circum-Medit. WBS.

Torilis nodosa (L.) Gaertn. T scap. Euro-Medit.Irano-Turan. WBS.

\section{ASTERACEAE}

Anacyclus clavatus (Desf.) Pers. T scap. CircumMedit. WBS.

Andryala integrifolia L. T scap. Circum-Medit. WBS.

Anthemis arvensis L. subsp. incrassata (Loisel.) Nyman. T scap. Circum-Medit. WBS.

Anthemis maritima L. H scap. W Medit. WRL.

Anthemis secundiramea Biv. T scap. C Medit. WRS.

Artemisia arborescens L. NP. Circum-Medit. WBL.

Asteriscus aquaticus (L.) Less. subsp. aquaticus. T scap. Circum-Medit. WBS.

Bellis annua L. subsp. annua. T scap. Medit.Atl. WBL.

Bellis perennis L. H ros. Euro-Medit. WBL.

**Bellium bellidioides L. H ros. Endem. SA-COBL. NRL.

Calendula arvensis L. T scap. Euro-Medit. WBS Carduus argyroa Biv. T scap. W Medit. WBS.

Carduus pycnocephalus L. subsp. pycnocephalus. $\mathrm{H}$ bienn. Medit.-Irano-Turan. WBS.

Carlina corymbosa L. H scap. W Medit. WBL.

Carlina lanata L. T scap. Circum-Medit. WBS.

Carlina racemosa L. T scap. SW Medit. WBS.

Carthamus caeruleus L. H scap. Circum-Medit. WBS.

Carthamus lanatus L. T scap. Medit.-IranoTuran. WBS.

Centaurea calcitrapa L. H bienn. Medit.-Atl. WBL.

Centaurea melitensis L. T scap. Circum-Medit. WBS.

Centaurea napifolia L. T scap. W Medit. WBS.

Centaurea sphaerocephala L. H scap. CircumMedit. WBS.

Chamaeleon gummifer (L.) Cass. H ros. S Medit. WBL. 
Chamaemelum fuscatum (Brot.) Vasc. T scap. W Medit. WBS.

Chondrilla juncea L. H scap. Euro-Medit.-IranoTuran. WBS.

Cichorium intybus L. H scap. Paleotemp. WBS.

Cirsium lanceolatum Hill. H bienn. Paleotemp. WBS.

Cirsium vulgare (Savi) Ten. $\mathrm{H}$ bienn. Paleotemp. WBS.

Cladanthus mixtus (L.) Oberpr. et Vogt. T scap. Circum-Medit. WBS.

Coleostephus myconis (L.) Cass. ex Rchb. $f$. T scap. Circum-Medit. WBS.

Crepis bellidifolia Loisel. T scap. W Medit. WBS. Crepis foetida L. H bienn. Paleotemp. WBS.

**Crepis vesicaria $\mathrm{L}$. subsp. hyemalis (Biv.) Ces., Pass. et Gib. T scap. Endem. SA-SI. NRS.

*Crepis vesicaria L. subsp. taraxacifolia (Thuill.) Thell. H scap. Euro-Medit.

Cynara cardunculus L. H scap. Circum-Medit. WBS.

Dittrichia graveolens (L.) Greuter. T scap. Medit.-Irano-Turan. WBL.

Dittrichia viscosa (L.) Greuter. H scap. W Medit. WBL.

Erigeron bonarensis L. T scap. Avv. (Neotrop.). WBS.

Erigeron canadensis L. T scap. Cosmop. WBS.

Erigeron sumatrensis Retz. T scap. Nat. (America Trop.). WBS.

Filago asteriscifolia (Lam.) Chrtek et Holub. T rept. Circum-Medit. WBS.

Filago gallica L. T scap. Euro-Medit. WBS.

Filago pygmaea L. T rept. Circum-Medit. WBS.

Filago pyramidata L. T scap. Euro-Medit. WBS.

Filago vulgaris Lam. T scap. Paleotemp. WBS.

Galactites elegans (All.) Soldano. H bienn. Circum-Medit. WBL.

Glebionis coronaria (L.) Spach. T scap. CircumMedit. WBS.

Glebionis segetum (L.) Fourr. T scap. CircumMedit. WBS.

Hedypnois cretica (L.) Dum. Cours. T scap. Circum-Medit. WBS.

Hedypnois rhagadioloides (L.) F.W. Schmidt. T scap. Circum-Medit. WBS.

**Helichrysum microphyllum (Willd.) Camb. subsp. tyrrhenicum Bacch., Brullo et Giusso. Ch suffr. Endem. SA-CO-BL. NRL.

Helminthotheca echioides (L.) Holub. T scap.
Circum-Medit. WRS.

Hypochaeris achyrophorus L. T scap. CircumMedit. WRL.

Hypochaeris glabra L. T scap. Euro-Medit. WRS.

*Lactuca muralis (L.) Gaertn. H scap. Europ.Caucas.

Leontodon tuberosus L. H ros. Circum-Medit. WBS.

Limbarda crithmoides (L.) Dumort. subsp. crithmoides. Ch suffr. Euro-Medit. WBS.

**Nananthea perpusilla (Loisel.) DC. T scap. Endem. SA-CO. NRS.

Notobasis syriaca (L.) Cass. T scap. CircumMedit. WBS.

Onopordum illyricum L. subsp. illyricum. H bienn. Circum-Medit. WBS.

Otanthus maritimus (L.) Hoffmanns. et Link subsp. maritimus. Ch suffr. Circum-Medit. WRL.

Pallenis spinosa (L.) Cass. subsp. spinosa. H bienn. Circum-Medit. WBS.

Phagnalon rupestre (L.) DC. subsp. annoticum (Jord. ex Burnat) Pignatti. Ch suffr. W Medit. WRS.

Pulicaria odora (L.) Rchb. H scap. CircumMedit. WRS.

Pulicaria sicula (L.) Moris. T scap. CircumMedit. WRS.

Reichardia picroides (L.) Roth. H scap. CircumMedit. WBS.

Rhagadiolus stellatus (L.) Gaertn. T scap. Circum-Medit. WBS.

Scolymus hispanicus L. H bienn. Euro-Medit. WBS.

Scolymus maculatus L. T scap. Circum-Medit. WBS.

Senecio lividus L. T scap. Circum-Medit. WBS.

**Senecio transiens (Rouy) Jeanm. T scap. Endem. SA-CO. NRS.

Senecio vulgaris L. T scap. Paleotemp. WBS.

Silybum marianum (L.) Gaertn. H bienn. Circum-Medit. WBS.

Sonchus asper (L.) Hill. T scap. Cosmop. WBS.

Sonchus bulbosus (L.) N. Kilian et Greuter subsp. bulbosus. G bulb. Circum-Medit. WBS.

Sonchus oleraceus L. T scap. Boreo-Trop. WBS.

Sonchus tenerrimus L. T scap. Circum-Medit. WBS.

Symphyotrichum squamatum (Spreng.) G.L. 
Nesom. T scap. Neotrop. WBS.

Tolpis umbellata Bertol. T scap. Circum-Medit. WBS.

Tolpis virgata (Desf.) Bertol. H scap. CircumMedit. WBS.

Tripolium pannonicum (Jacq.) Dobrocz. subsp. tripolium (L.) Greuter. H bienn. Euro-Medit.Irano-Turan. WBS.

Urospermum dalechampii (L.) F.W. Schmidt. H scap. Circum-Medit. WBL.

Urospermum picroides (L.) Scop. ex F.W. Schmidt. T scap. Circum-Medit. WBS.

Xanthium orientale L. subsp. italicum (Moretti) Greuter. T scap. Nat. (America). WBS.

Xanthium spinosum L. T scap. Avv. (Sudamer.). WBS.

CAMPANULACEAE

Campanula erinus L. T scap. Circum-Medit. WBS.

Legousia hybrida (L.) Delabre. T scap. EuroMedit. WBS.

\section{DIPSACACEAE}

Centranthus calcitrapae (L.) Dufr. subsp. calcitrapae. T scap. Circum-Medit. WBS.

**Dipsacus ferox Loisel. H bienn. Endem. SACO. NBS.

*Dipsacus fullonum L. H bienn. Euro-Medit.

Lonicera implexa Aiton subsp. implexa. P lian. Circum-Medit. WBS.

Pycnocomon rutifolium (Vahl) Hoffmanns. et Link. T scap. W Medit. WRL.

Sixalix atropurpurea (L.) Greuter et Burdet subsp. maritima (L.) Greuter et Burdet. H bienn. Circum-Medit. WBS.

Valerianella dentata (L.) Pollich. T scap. EuroMedit. WBS.

Valerianella microcarpa Loisel. T scap. CircumMedit. WBS.

Taxa la cui posizione tassonomica non è ancora definita:

CYNOMORIACEAE

Cynomorium coccineum L. subsp. coccineum. G rhiz. Medit.-Irano-Turan. WRS.

RAFFLESIACEAE

Cytinus hypocistis (L.) L. G rad. Circum-Medit.
WRS.

Cytinus ruber Fourr. ex Fritsch. G rad. CircumMedit. WRS.

\section{DISCUSSIONE E CONCLUSIONI}

La flora del Sinis risulta costituita da 760 unità tassonomiche di cui 615 di rango specifico, 134 subspecifico, 10 varietale e 1 ibrido, appartenenti a 87 famiglie e 365 generi. Tra le Angiospermae, le Eudicots costituiscono il gruppo sistematico di gran lunga più numeroso con 549 taxa, riferibili a 57 famiglie e 259 generi; le Monocots contano invece 198 entità, appartenenti a 20 famiglie e 96 generi. Le Pteridophyta e le Gymnospermae, con 4 e 5 taxa, rappresentano rispettivamente lo $0,53 \%$ e lo $0,66 \%$ della flora.

Tra le famiglie con il maggior numero di entità troviamo le Poaceae (99), seguite dalle Fabaceae e dalle Asteraceae (85); sono inoltre significativi i valori delle Caryophyllaceae (33), delle Apiaceae (27) e delle Orchidaceae (24).

Tra i generi con il maggior numero di entità troviamo Trifolium (19), Silene (14), Limonium e Medicago (13), Ophrys (12), Euphorbia e Vicia (10), Plantago (9), Allium (8) e infine Lotus, Ranunculus e Vulpia (7).

I dati dello spettro biologico confermano la mediterraneità dell'area con un valore molto elevato di terofite $(\mathrm{T}=48,81 \%)$; questo dato sottolinea chiaramente l'accentuata e prolungata xericità della zona, associata a valori di temperatura media elevati. Ciò risulta in linea con i dati di altre aree costiere della Sardegna, nelle quali la ventosità, le alte temperature e quindi l'elevata evapotraspirazione determinano la massiccia presenza di specie a ciclo annuale. L'elevato valore delle terofite indica inoltre la presenza significativa di ambienti sinantropici o 
disturbati, dove si assiste ad una esplosione di specie effimere ad areale di distribuzione ampio.

Alle condizioni climatiche dell'area è anche correlato il valore delle camefite $(8,03 \%)$; l'elemento che influisce su tale aumento è il vento, tipicamente da NordOvest o Ovest. La percentuale di geofite $(12,76 \%)$ appare invece legata all'uso antropico del territorio, tale dato offre una chiara indicazione sullo stato di degrado dell'area, determinato dall'utilizzazione a scopi agricoli dei terreni e secondariamente dalla pratica degli incendi.

E' interessante notare come il dato relativo alle idrofite si mantenga relativamente elevato $(2,24 \%)$, per effetto della presenza di numerosi stagni retrodunali lungo tutta l'area e di pozze temporanee, molto diffuse soprattutto nel plateau basaltico di Capo San Marco.

Risulta molto basso il dato relativo alle fanerofite $(5,13 \%)$, in ragione del fatto che le formazioni forestali sono praticamente assenti e confinate in piccole aree marginali, mentre la quasi totalità del territorio è ampiamente utilizzata per le colture agrarie e per le attività zootecniche. L'unica vera eccezione è rappresentata dalla pineta artificiale di Is Arenas, impiantata tra il 1950 e il 1957 nell'ambito della realizzazione dei lavori di sistemazione idraulico-forestale finalizzati a limitare la movimentazione delle sabbie e favorire 1'utilizzo agricolo dei terreni retrostanti. Tale imboschimento si estende lungo una fascia litoranea di circa 8 $\mathrm{Km}$ e occupa una superficie di circa 1100 ettari.

Per gli aspetti corologici risulta dominante il gruppo delle specie mediterranee $(79,21 \%)$, rispetto a tutte le altre componenti corologiche generali che oscillano, in maniera assai limitata, tra valori variabili dal 3,42 al 6,05\%.

L'analisi corologica delle specie a gravitazione mediterranea evidenzia la dominanza dei subelementi circummediterranei con 270 unità, seguiti da quelli euromediterranei (83) e dalle entità endemiche (54). Interessante il dato relativo alle specie a gravitazione mediterraneoatlantica (43) e ovest-mediterranea (75), che forniscono importanti indicazioni rispetto alla correlazione tra la flora della placca Sardo-Corsa e quella dei territori Catalani e Provenzali, a testimonianza degli antichi legami paleogeografici; tutto ciò conferma anche l'inquadramento biogeografico dei territori sardi all'interno della subregione biogeografica del Mediterraneo occidentale.

Per quanto concerne la componente endemica, si è ritenuto opportuno elaborare $i$ dati in maniera più particolareggiata poiché la Sardegna, principalmente a causa dell'insularità, risulta ricca di taxa endemici, tanto da essere considerata come uno dei principali hot spot di biodiversità del Mediterraneo (Médail, Quézel, 1997). Le indagini condotte hanno permesso di censire 54 endemiti (tab. 3), 31 sono risultati di rango specifico, 17 sottospecifico e 6 varietale, suddivisi in 22 famiglie e 38 generi.

Tra le famiglie più ricche in endemismi troviamo le Plumbaginaceae (10), seguite da Asteraceae e Lamiaceae (5), Fabaceae (4), Alliaceae, Euphorbiaceae e Ranunculaceae (3).

Il genere che in assoluto presenta il maggior numero di unità tassonomiche endemiche è Limonium (10); seguono poi altri sei generi con 2 taxa ciascuno: Allium, Delphinium, Euphorbia, Scrophularia, Silene e Teucrium. Il dato delle Plumbaginaceae, e più nello specifico del genere Limonium, appare certamente connesso sia alla grande estensione costiera del Sinis, sia alla notevole diversità litologica e alla dominanza di coste rocciose.

L'analisi delle forme biologiche mostra 


\begin{tabular}{|c|c|c|c|}
\hline Unità tassonomica & ma biologica & Forma corologica & Unità biogeografica \\
\hline Allium parciflorum Viv. & G bulb & Endem. SA-CO & Provincia Sardo-Corsa \\
\hline Allium roseum $\mathrm{L}$. var. insulare Gennari & G bulb & Endem. SA-CO & Provincia Sardo-Corsa \\
\hline Anchusa littorea Moris & T scap & Endem. SA & Subprovincia Sarda \\
\hline Arum pictum L. $f$. subsp. pictum & G rhiz & Endem. SA-CO & Provincia Sardo-Corsa \\
\hline $\begin{array}{l}\text { Astragalus pelecinus (L.) Barneby } \\
\text { subsp. pelecinus }\end{array}$ & T scap & 1. SA-CO & perprovincia Italo-Tirren \\
\hline Bellium bellidioides L. & H ros & Endem. SA-C & Subregione Mediterraneo-occidentale \\
\hline $\begin{array}{l}\text { Centaurium erythraea Rafn. } \\
\text { subsp. rhodense (Boiss. et Reut.) Melderis } \\
\text { Crepis vesicaria } \mathrm{L} \text {. }\end{array}$ & T scap & Endem. SA-CO-SI-ITM & Superprovincia Italo-Tirrenica \\
\hline subsp. hyemalis (Biv.) Ces., Pass. et Gib. & T scap & Endem. SA-SI & Superprovincia Italo-Tirrenica \\
\hline Crocus minimus DC. & G bulb & Endem. SA-CO-AT & Superprovincia Italo-Tirrenica \\
\hline Delphinium longipes Moris & T scap & Endem. SA & Subprovincia Sarda \\
\hline Delphinium pictum subsp. pictum Willd. & H scap & Endem. SA-CO-BL-H & Subregione Mediterraneo-occidentale \\
\hline $\begin{array}{l}\text { Dipsacus ferox Loisel. } \\
\text { Euphorbia pithyusa L. }\end{array}$ & H bienn & Endem. SA-CO & Provincia Sardo-Corsa \\
\hline subsp. cupanii (Guss. ex Bertol.) Radel-Sm. & Ch suffr & Endem. SA-CO-SI & Superprovincia Italo-Tirrenica \\
\hline $\begin{array}{l}\text { Euphorbia pithyusa L. subsp. pithyusa } \\
\text { Ferula arrigonii Bocchieri }\end{array}$ & $\begin{array}{l}\text { Ch suffr } \\
\text { H scap }\end{array}$ & Endem. SA-CO-AT-BL & Subregione Mediterraneo-occidentale \\
\hline Galium verrucosum Huds. & & & \\
\hline var. halophilum (Ponzo) Natali et Jeanmonod & d T scap & Endem. SA-CO-AT-SI & Superprovincia Italo-Tirrenica \\
\hline Genista corsica (Loisel.) DC. & NP & Endem. SA-CO & Provincia Sardo-Corsa \\
\hline $\begin{array}{l}\text { Helichrysum microphyllum (Willd.) Camb. } \\
\text { subsp. tyrrhenicum } \text { Bacch., Brullo et Giusso } \\
\text { Limonium acutifolium (Rchb.) Salmon }\end{array}$ & Ch suffr & Endem. SA-CO-BL & Subregione Mediterraneo-occidentale \\
\hline subsp. acutifo & Ch suffr. & Endem. SA-CO & Provincia Sardo-Corsa \\
\hline Limonium capitis-marci Arrigoni et Diana & Ch suffr & Endem. SA & Sottosettore Sinisico \\
\hline Limonium cornusianum Arri & Ch suffr & Endem. SA & Subprovincia Sarda \\
\hline Limonium dubium (Andr. ex Guss.) Litard. & $\mathrm{H}$ ros & Endem. SA-CO-SI & Superprovincia Italo-Tirrenica \\
\hline Limonium glomeratum (Tausch) Erben & Ch suffr & Endem. SA-SI & Superprovincia Italo-Tirrenica \\
\hline Limonium lausianum Pignatti & Ch suffr & Endem. SA & Sottosettore Sinisico \\
\hline Limonium oristanum May & Ch suffr & Endem. SA & Sottosettore Sinisico \\
\hline Limonium pseudolaetum Arrigoni et Diana & Ch suffr & Endem. SA & Sottosettore Sinisico \\
\hline Limonium tenuifolium (Bertol. ex Mori & nCh suffr & Endem. SA & Sottosettore Sinisico \\
\hline Limonium tharrosianum Arrigon & Ch suffr & Endem. SA & Sottosettore Sinisico \\
\hline $\begin{array}{l}\text { Lotus cytisoides L. subsp. conradiae Gamisans } \\
\text { Medicago intertexta (L.) Mill. }\end{array}$ & Ch suff & Endem. SA-CO & Provincia Sardo-Corsa \\
\hline var. tubers & T scap & Enc & Sarda \\
\hline Mercurialis corsica Coss. et Kralil & Ch suffr & Endem. SA-CO & Provincia Sardo-Corsa \\
\hline $\begin{array}{l}\text { Micromeria filiformis (Aiton) Benth. } \\
\text { subsp. filiformis }\end{array}$ & Ch suff & En & Subre \\
\hline Nananthea perpusilla (Loisel.) DC. & & Enden & Provincia Sardo-Corsa \\
\hline Ophrys morisii (Martelli) Soò & G bulb & Endem. SA-CO & Provincia Sardo-Corsa \\
\hline $\begin{array}{l}\text { Ophrys subfusca (Rchb. } f \text {.) Hausskn. } \\
\text { subsp. liveranii G. Orrù et M.P. Gras }\end{array}$ & ull & & \\
\hline rsicum Jord. et Fourr. & G bulb & Ende & $\begin{array}{l}\text { Subprovinc1 } \\
\text { Provincia Sarc }\end{array}$ \\
\hline Pancratium ill & G bulb & A-CO-AT & Iperprovincia Italo-Tirrenica \\
\hline Phillyrea media L. var. rodriguezii $\mathrm{P}$. Monts. & $P$ caesp & Endem SA-CO-BL & Subregione Mediterraneo-occidentale \\
\hline Polyg & Ch suffr & Endem. SA & Sottosettore Sinisico \\
\hline $\begin{array}{l}\text { Polygonum scoparium Req. ex Loisel. } \\
\text { Prospero autumnale (L.) Speta }\end{array}$ & Ch frut & Endem. SA-CO & Provincia Sardo-Corsa \\
\hline var. corsica (Boullu) Bri & G bulb & Endem. SA-CO & Provincia Sardo-Corsa \\
\hline Ranunculus cordiger Viv. & & & \\
\hline oris) Arrigoni & H scap & Enden & Corsa \\
\hline Romulea requienii Parl. & G bulb & Endem. SA-C & Provincia Sardo-Corsa \\
\hline $\begin{array}{l}\text { var. palaui } \mathrm{O} \text {. Bolos et } \mathrm{R} \text {. Mol. } \\
\text { Rubia peregrina } \mathrm{L} \text {. subsp. requienii }\end{array}$ & NP & Endem. SA-CO- & Subregione Mediterraneo-occidentale \\
\hline (Duby) Cardona $e$ & P lian & Enden & SL \\
\hline Scrophularia ramosissima Loisel. & Ch suffr & Endem. SA-CO-BL-GA & Subregione Mediterraneo-occidentale \\
\hline Scrophularia & H scap & Endem. SA-CO-AT & Superprovincia Italo-Tirrenica \\
\hline (Rouy) Jeanm. & cap & Ende & Provincia Sardo-Corsa \\
\hline Silene beguinotii Vals. & T scap & Endem. SA & Subprovincia Sarda \\
\hline $\begin{array}{l}\text { Silene succulenta Forssk. } \\
\text { subsp. corsica (DC.) Nyman }\end{array}$ & & & \\
\hline Stachys glutinosa L. & Ch frut & Endem. SA-CO-AT & Superprovincia Italo-Tirrenica \\
\hline Teucrium marum L. subsp. marum & Ch frut & Endem. SA-CO-AT-BL-H & Subregione Mediterraneo-occidentale \\
\hline Teucrium subspinos & Ch frut & End & gione Medi \\
\hline trovirens $\mathrm{Re}$ & H scap & Endem. SA-CO-AT & Superprovincia Italo-Tirrenica \\
\hline
\end{tabular}


la prevalenza delle camefite ( 21 pari al $39,63 \%)$, seguite dalle emicriptofite $(10=$ $18,87 \%)$, secondariamente delle terofite e delle geofite $(9=16,98)$, mentre le fanerofite e le nanofanerofite rappresentano entrambe il $3,77 \%$ con 2 unità tassonomiche rispettivamente. Si osserva che il valore delle camefite risulta molto più alto rispetto $\mathrm{a}$ quello rilevato per lo spettro biologico generale dell'intera flora $(8,04 \%)$ e questo deve essere ricondotto alla elevatissima diversità specifica rappresentata dal genere Limonium.

Lo spettro biologico limitato alla componente endemica registra un netto calo delle terofite col 16,98\% rispetto al $48,48 \%$ dello spettro generale, confermando quanto già rilevato per aree limitrofe da Bacchetta et Pontecorvo (op. cit.). Ciò avvalora il fatto che la gran parte degli endemiti non si adattano con facilità agli ambienti sinantropici o disturbati, dove invece si assiste ad una esplosione di specie ad areale di distribuzione ampio. Va a questo proposito rilevato come la gran parte dei taxa endemici terofitici siano al contrario caratteristici di habitat altamente selettivi, quali quelli psammofili, a conferma dell'elevato grado di specializzazione di tali unità tassonomiche. In particolare merita sottolineare il fatto che diverse specie terofitiche degli ambiti dunali sono intolleranti a qualsiasi fenomeno di modificazione e disturbo di tali habitat (Bacchetta, 2006).

Dal punto di vista corologico tra gli endemismi prevalgono gli elementi della provincia biogeografica Sardo-Corsa $(33,33 \%)$ e secondariamente quelli della sottoprovincia Sarda $(24,10 \%)$, delle isole del Mediterraneo Occidentale (18,52\%), delle isole Tirreniche $(14,80 \%)$, dell'area Tirrenica comprensiva delle coste italiche $(5,55 \%)$ e, infine le entità Sardo-Sicule $(3,70 \%)$. Questi dati confermano l'inquadramento biogeografico dell'area nella regione biogeografica Mediterranea e più nello specifico nella subregione del Mediterraneo occidentale, superprovincia Italo-Tirrenica, provincia Sardo-Corsa e subprovincia Sarda (Ladero Alvarez et al., 1987; Bacchetta, Pontecorvo, op. cit.).

Tra le unità tassonomiche endemiche rivestono particolare importanza le 6 specie del genere Limonium esclusive del Sinis (Limonium capitis-marci, L. lausianum, L. tharrosianum, L. oristanum, L. pseudolaetum e L. tenuifolium), che confermano l'elevata diversità ed il pregio naturalistico dell'area indagata. In particolare, si sottolinea la presenza di L. pseudolaetum, specie alofila esclusiva del Sinis, che vegeta ai margini delle depressioni umide retrodunali e risulta inserita negli allegati della Diretiva Habitat 92/43/CEE come specie prioritaria.

Oltre alle suddette specie, risulta esclusiva del Sinis anche Polygala sinisica, endemita presente solo nel promontorio di Capo Mannu, dove è stata censita nel 2007 una ridottissima popolazione di circa 70 individui riproduttori. La specie appare assai rara e a forte rischio di estinzione anche per la progressiva scomparsa degli habitat in cui si rinviene, determinata principalmente dalle attività antropiche di trasformazione e utilizzo del territorio che insistono sull'area. Sulla base dei nuovi dati $P$. sinisica, inserita tra le 50 specie del Mediterraneo a maggior rischio di estinzione (Montmollin De, Strahm, 2005), secondo la categorie IUCN e le recenti indicazioni metodologiche (IUCN, 2006) viene catalogata come CR [B1ab(ii)+2ab(ii)].

Non considerata nella suddetta pubblicazione ma, in grave pericolo d'estinzione risulta essere Anchusa littorea, specie non ritrovata durante la presente indagine e la cui ultima segnalazione per il Sinis, riferita all'area di Is Arenas, risale ai primi anni 80 (Valsecchi, 1980; Brambilla 
et al., 1982). La distribuzione attuale di questa specie, ritenuta estinta sino a poco tempo fa (Bacchetta, Pontecorvo, op. cit.) è limitata all'area di Scivu-Piscinas (Arbus), dove è presente una piccola popolazione che presenta oscillazioni demografiche consistenti interannuali (Bacchetta et al., 2007).

Riveste un rilevante interesse Micromeria filiformis subsp. filiformis, endemica delle Isole Baleari (Mallorca, Minorca e Ibiza) e della Sardegna, dove risulta presente solo nella Penisola del Sinis con tre stazioni (Mulas, 1984; Arrigoni, 1986), che possono essere considerate facenti parte di un'unica popolazione in quanto non esistono barriere fisiche e genetiche. Tale entità veniva considerata sino a pochi anni fa endemica anche della Corsica, più recentemente però Kerguélen (1987) ha rivalutato la var. minutilifolia Chodat elevandola al rango di sottospecie e considerandola endemica esclusiva della Corsica (Gamisans, Marzocchi, 1996), così come risulta essere esclusiva dei calcari mesozoici della Sardegna centro-orientale $M$. filiformis subsp. cordata (Bertol.) Pignatti (Conti et al., 2005).

Merita una particolare citazione anche la presenza di Nananthea perpusilla che, nel Sinis, si rinviene solo nella parte occidentale dell'Isola di Mal di Ventre. Nell'isola si rinvengono popolamenti omogenei, anche di estensione notevole, in situazioni dove si creano tasche di suolo ricco in materia organica e con elevato tenore in umidità durante il periodo primaverile. La specie risulta meritevole di conservazione poichè considerata un paleoendemismo con areale relitto che presenta una graduale riduzione nelle aree più settentrionali (Corrias, 1977; Corrias, 1981).

Tra le endemiche merita anche segnalare il ritrovamento di Delphinium longipes, specie descritta dal Moris per le sabbie marittime di Pula e a lungo inclusa in $D$. halteratum $\mathrm{Sm}$. Tale entità, solo di recente rivalutata per la Sardegna (Bocchieri, Mulas, op. cit.; Bacchetta, Pontecorvo, op. cit.; Bacchetta, op. cit.), segnalata da Bocchieri et Mulas (op. cit.) a Capo S. Marco, in un numero esiguo di individui, è stata rinvenuta anche sulle sabbie attorno alla laguna di Mistras dove è presente una consistente popolazione.

Rivestono particolare importanza i ritrovamenti di Rosmarinus officinalis var. palaui e Phillyrea media var. rodriguezii, in quanto varietà endemiche delle isole del Mediterraneo occidentale finora non segnalate per la Sardegna e che quindi risultano nuove per la flora italiana.

Tra le nuove segnalazioni per l'area merita un cenno il ritrovamento di Rubia peregrina subsp. requienii, endemismo tirrenico già segnalato per il Guspinese da Cardona et Sierra-Ràfols (1981). Questo nuovo ritrovamento, conferma la presenza del taxon nell'Isola e amplia l'areale di distribuzione sino ad oggi considerato limitato al settore biogeografico SulcitanoIglesiente.

Per quanto concerne le specie d'interesse fitogeografico, lo studio condotto ha permesso di segnalare nuove entità per la Sardegna e confermare numerose specie di cui si apportano dati aggiornati relativi all'areale di distribuzione e alla consistenza delle popolazioni.

In particolare viene segnalata come nuova per la flora sarda Arundo collina, rinvenuta nella parte settentrionale di Is Arenas, lungo il Rio Pischinappiu, e nei territori di Riola, Nurachi e Cabras, nei pressi di piccoli corsi d'acqua. Questo ritrovamento consente di ampliare l'areale della specie che in Italia, secondo quanto riportato da Danin et al. (2005), veniva data per la parte centromeridionale della penisola e per la Sicilia.

Riveste grande importanza il 
ritrovamento di Lagurus ovatus subsp. vestitus, mai segnalato prima per la Sardegna e rinvenuto in un'unica popolazione di ridotte dimensioni nella parte meridionale del campo dunare di Is Arenas. Questa segnalazione contribuisce a meglio definire la distribuzione di tale sottospecie, ad oggi conosciuta in Italia esclusivamente per la Sicilia (Conti et al., op. cit.).

Una citazione particolare merita Helianthemum caput-felis, specie inserita negli Allegati della Direttiva 92/43/CEE, segnalata nella parte più settentrionale del Sinis, nella zona compresa tra Is Aruttas e Capo Mannu e costituente l'unica popolazione per l'Italia (Arrigoni, 1971; Pignatti et al., op. cit.). Nel corso degli studi di carattere biologico conservativo, è stato possibile rinvenire alcuni esemplari della specie anche lungo le falesie mioceniche del promontorio di Seu, ampliando quindi l'areale della popolazione finora conosciuto. Tale stazione risulta fortemente minacciata per effetto della evoluzione geomorfologica della falesia, nella quale si sono verificati ripetuti crolli e cedimenti. Allo stesso modo i nuclei presenti lungo le falesie di Su Tingiosu sono costituiti da pochi individui, relegati in una fascia di 1-2 metri tra la falesia e la nuova strada panoramica; anche in quest'area si segnalano negli ultimi anni numerosi crolli e frane che, in assenza di interventi di conservazione adeguati, potrebbero comportare una riduzione del numero di individui e dell'areale della specie in Sardegna.

Di particolare interesse è la presenza di Viola arborescens, già segnalata per la parte settentrionale del promontorio di Seu (Mulas, 1993) e Capo Mannu (Arrigoni, 1972; Bocchieri et al., op. cit.), ritrovata oggi anche a Is Aruttas e in tutta l'area compresa tra Capo Mannu, Su Pallosu e Su Crastu Biancu. Un ulteriore popolamento, costituito da 40 individui riproduttori, è stato ritrovato nella parte meridionale del promontorio di Seu, ai margini del sentiero che porta alla spiaggia di Abbarossa, il che consente di ampliare ulteriormente l'areale di distribuzione della specie. Queste stazioni, insieme a quelle segnalate nel Nord-Ovest della Sardegna per il sassarese (Nicotra, 1898; Atzei, 1981; Bagella, Urbani, 2006) e Alghero (Valsecchi, 1981), sono le uniche note per la flora italiana. La presenza della specie in diverse località lungo la costa occidentale potrebbe essere indicativa di una più ampia distribuzione lungo le aree costiere della Sardegna occidentale.

Si conferma la presenza di Gennaria diphylla, segnalata per diverse località del nord Sardegna (Gennari, 1870; Barbey, 1885; Vaccari, 1894, 1896; Desole 1956, 1961; Bocchieri, 1996), per la pineta di Is Arenas (Giotta, Piccitto, 1995) e per l'Iglesiente presso Fluminimaggiore (Scrugli, Cogoni, 1995). Della specie è stata rinvenuta una importante popolazione, all'interno della pineta di Is Arenas, dove sono presenti diverse migliaia di individui su una superficie di circa 20 ettari. Inoltre sono stati ritrovati individui isolati nelle garighe costiere calcicole presso la località di Sa Mesa Longa; tale ritrovamento appare di elevato interesse in considerazione del fatto che la specie non risulta segnalata su substrati carbonatici.

Altra specie d'interesse fitogeografico, la cui presenza in Italia appare limitata a pochissime località tirreniche costiere della Liguria, Toscana, Campania, Calabria e Sicilia, è Coris monspeliensis subsp. monspeliensis. Questo taxon in Sardegna risulta esclusivo della Penisola del Sinis, dove è stato osservato solo a Seu, soprattutto nella parte più settentrionale del promontorio dove si incontra la popolazione più consistente, e a Capo Mannu, dove risulta più sporadico a causa degli interventi di forestazione realizzati negli anni ottanta. 
Particolare attenzione merita Quercus calliprinos, specie del Mediterraneo centroorientale che in Sardegna trova il limite occidentale del suo areale; il comportamento di questa specie in Sardegna è stato oggetto di diversi studi di carattere tassonomico e vegetazionale, soprattutto per il fatto che non si accompagna ad altre entità tipiche del Mediterraneo orientale (Mossa, 1990). Nel Sinis gli individui di quercia spinosa si ritrovano esclusivamente nell'area dunale di Is Arenas e costituiscono i popolamenti piu settentrionali della specie in Sardegna. In tale area non si osservano formazioni forestali, probabilmente a causa del degrado e della frammentazione degli habitat forestali psammofili determinata dai lavori che portarono alla piantumazione della pineta artificiale.

Tra le specie d'interesse fitogeografico a gravitazione occidentale, meritano un cenno Armeria pungens ed Ephedra distachya. La prima risulta segnalata da Bocchieri et al. (op. cit.) per le zone sabbiose e le zone retrodunali della parte più settentrionale dell'area di Capo Mannu, dove oggi rimangono solo pochi individui per effetto delle modificazioni legate alle attività turistiche di tipo balneare che hanno subito gli habitat psammofili. La seconda risulta gravemente minacciata in tutta Italia e segnalata in Sardegna solo per i sistemi dunali della Costa Verde compresi tra Scivu e Pistis e alcune aree della fascia costiera Nord-Occidentale. Nonostante la specie sia apparentemente diffusa nei territori di studio, è in pericolo e in forte regressione se si considera che circa 40 anni fa occupava “. aree vaste anche un ettaro e più. " (Desole, 1965), mentre attualmente appare circoscritta a piccoli nuclei fortemente frammentati. Gli interventi di bonifica a scopo agricolo, hanno portato a una drastica riduzione dell'habitat di questa specie, per la quale appare necessario impostare una adeguata strategia di conservazione.

Per quanto concerne le forme di rarità di Rabinowitz si evidenzia la dominanza delle entità riferite alla categoria WBS $(326=44,29 \%)$, seguite dalle WBL $(119=16,17 \%)$, WRS $(105=14,27 \%)$ e WRL $(85=11,55 \%)$; in subordine si trovano invece le restanti quattro categorie con valori oscillanti tra il 2,72 ed il 3,94\%.

L'analisi dettagliata delle componenti evidenzia come 101 unità tassonomiche $(13,72 \%)$ su un totale di 736 alle quali è stato possibile assegnare un indice, sono ad areale ristretto (NRS, NRL, NBS, NBL). Emerge inoltre il dato considerevole delle unità tassonomiche ad elevata specificità ecologica (236 taxa, pari al 32,06\%), riferite alle categorie NRS, NRL, WRS e WRL. Questi dati confermano quanto già esposto in merito alla presenza di habitat estremamente selettivi, quali quelli psammofili, rupicoli ed alofili; il tutto permette inoltre di enfatizzare l'effetto di diversi fattori limitanti quali l'aerosol marino, la ventosità e la prolungata aridità estiva.

Un ulteriore dato interessante riguarda la taglia delle popolazioni presenti; in particolare ben 483 unità tassonomiche $(65,62 \%)$, riferite alle categorie NRS, NBS, WRS e WBS, presentano popolazioni di dimensioni ridotte a parziale conferma dell'autonomia floristica della Penisola del Sinis rispetto ai territori circostanti, considerazione che permette di avvalorare ulteriormente l'individuazione di un sottosettore biogeografico indipendente.

In ultimo si fornisce un elenco (tab. 4) di tutti i taxa inseriti nelle liste rosse regionali d'Italia (Conti et al., 1997), recentemente riviste da Scoppola et Spampinato (op. cit.). Per tutte le endemiche esclusive e per quelle di notevole importanza conservazionistica a livello regionale, vengono altresì proposte le nuove categorie 
di protezione, elaborate secondo i criteri e le linee guida stabiliti dalla IUCN (1994; 2001 ; 2003; 2006) e sulla base del lavoro realizzato per l'endemoflora dell'Iglesiente (Bacchetta, Pontecorvo, op. cit.).

Dalla tabella 4 emerge che nelle liste rosse regionali del 1997 sono presenti 40 unità tassonomiche, di cui $2 \mathrm{CR}$ (gravemente minacciate), 3 EN (minacciate), $12 \mathrm{VU}$ (vulnerabili), $20 \mathrm{LC} / \mathrm{LR}$ (a minor rischio) e 3 per le quali non si hanno dati sufficienti (DD). Sulla base delle liste rosse nazionali aggiornate da Scoppola et Spampinato (op. cit.), vengono incluse invece 28 unità tassonomiche; in particolare le specie gravemente minacciate salgono a 5,4 risultano minacciate, 11 vulnerabili, 7 a minor rischio e per 1 sola unità tassonomica non si hanno sufficienti informazioni.

Le analisi condotte hanno permesso di verificare il reale stato di minaccia per tutte le specie della flora ed in particolare per quelle inserite nelle liste rosse regionali e nazionali. In base a ciò sono state modificate le categorie per 10 specie ed elaborate secondo i criteri della IUCN (2006), quelle relative a 3 specie precedentemente non considerate (Limonium cornusianum, $L$. oristanum e Micromeria filiformis subsp. filiformis). Sulla base delle modifiche effettuate si evidenzia come il numero di unità tassonomiche gravemente minacciate sia considerevolmente aumentato e oggi pari a 12; viene inoltre sottolinaeta la presenza in tale gruppo della gran parte dei taxa endemici esclusivi della Penisola del Sinis.

Il presente studio ha consentito di definire $i$ territori indagati, oltrechè dal punto di vista floristico, dal punto di vista biogeografico. Emerge, infatti, da un lato una ricca componente di unità tassonomiche esclusive dell'area che contribuiscono a differenziare il territorio da quelli circostanti; da un altro la significativa presenza percentuale di taxa comuni con i territori insulari del Mediterraneo occidentale, che consentono di inquadrare l'area a livello di provincia (Sardo-Corsa) e subregione biogeografica (Mediterranea occidentale). Per una completa analisi che consenta di individuare un settore biogeografico a cui riferire il Sinis, si ritiene comunque necessario completare le indagini nei restanti territori del Campidano. In prima analisi si considera che quanto proposto da Bacchetta et Pontecorvo (op. cit.) per il SulcisIglesiente, possa vedere una conferma della presenza di un settore biogeografico Campidanese che si estende lungo tutto il graben del Campidano, dal Golfo degli Angeli sino a quello di Oristano. In tale ottica certamente è possibile fin d'ora individuare, all'interno di un settore Campidanese, un sottosettore Sinisico, sulla base dei criteri proposti da Rivas-Martínez et al. (1999; 2002). Tale sottosettore si differenzia dai restanti territori del Campidano per la presenza di unità geolitologiche esclusive quali la Formazione di Capo San Marco, i calcari laminati del Sinis, la Formazione di Torre del Sevo e la Formazione di Capo Mannu, una morfologia peculiare e in grado di distinguere la Penisola del Sinis dai restanti territori pianeggianti e lagunari del Golfo di Oristano, oltre a condizioni di oceanicità climatica, testimoniate anche da una componente vegetale del tutto assente nei territori limitrofi (es. Gennaria diphylla). Dal punto di vista della diversità vegetale, la presenza di ben 6 specie endemiche esclusive e di numerosi taxa d'interesse fitogeografico a gravitazione occidentale (es. Micromeria filiformis subsp. filiformis, Coris monspeliensis subsp. monspeliensis, Helianthemum caput-felis, Lagurus ovatus subsp. vestitus, Phillyrea media var. rodriguezii, Rosmarinus officinalis var. palauii), presenti in Sardegna solo per i territori indagati, dimostrano l'elevato grado di autonomia floristica e giustificano ancor 


\begin{tabular}{|c|c|c|c|}
\hline BINOMIO SCIENTIFICO & IUCN 1997 & CN 200 & IUCN PROPOSTA \\
\hline Althenia filiformis Petit subsp. filiformis & LR (LC) & VU & \\
\hline Anchusa littorea Moris & EN & $\mathrm{CR}$ & $\mathrm{CR}[\mathrm{B} 1 \mathrm{ab}(\mathrm{i}, \mathrm{ii}, \mathrm{iii}, \mathrm{v})+2 \mathrm{ab}(\mathrm{i}, \mathrm{ii}, \mathrm{iii}, \mathrm{v})]$ \\
\hline Armeria pungens (Link) Hoffmanns. et Link & VU & & \\
\hline Atriplex rosea $\mathrm{L}$. & VU & & \\
\hline Bassia hirsuta (L.) Asch. & & VU & \\
\hline $\begin{array}{l}\text { Centaurium pulchellum (Sw.) Druce } \\
\text { subsp. pulchellum }\end{array}$ & DD & & \\
\hline Ceratophyllum demersum $\mathrm{L}$. & VU & & \\
\hline $\begin{array}{l}\text { Coris monspeliensis L. subsp. monspeliensis } \\
\text { Crepis vesicaria L. }\end{array}$ & VU & & EN [B1 ab(i, ii, iii, v) + 2ab(i, ii, iii, v)] \\
\hline subsp. hyemalis (Biv.) Ces., Pass. et Gib. & & DD & \\
\hline Cressa cretica $\mathrm{L}$. & & EN & \\
\hline Cymodocea nodosa (Ucria) Asch. & DD & & \\
\hline Cynomorium coccineum $\mathrm{L}$. & LR (LC) & VU & \\
\hline Cynosurus cristatus L. & EN & & \\
\hline Delphinium longipes Moris & & & EN [B1ab(i, ii, iii, iv, v) + 2ab(i, ii, iii, iv, v)] \\
\hline Delphinium pictum Willd. subsp. pictum & LR (LC) & & \\
\hline Elatine macropoda Guss. & & $\mathrm{CR}$ & \\
\hline Ephedra distachya $\mathrm{L}$. & & VU & $\mathrm{CR}[\mathrm{Alc}+2 ; \mathrm{B} 1 \mathrm{ab}(\mathrm{i}, \mathrm{ii}, \mathrm{iii}, \mathrm{iv}, \mathrm{v})+2 \mathrm{ab}(\mathrm{i}, \mathrm{ii}, \mathrm{iii}, \mathrm{iv}, \mathrm{v})]$ \\
\hline Eryngium barrelieri Boiss. & LR (LC) & & \\
\hline Euphorbia lathyris L. & LR (LC) & & \\
\hline Ferula arrigonii Bocchieri & LR (LC) & LR & \\
\hline Gennaria diphylla (Link) Parl. & VU & VU & \\
\hline Geranium robertianum $\mathrm{L}$. & VU & & \\
\hline Helianthemum caput-felis Boiss. & LR (LC) & LR & $\mathrm{VU}[\mathrm{A} 1 \mathrm{c}+2 ; \mathrm{B} 1 \mathrm{ab}(\mathrm{i}, \mathrm{ii}, \mathrm{iii}, \mathrm{iv})+2 \mathrm{ab}(\mathrm{i}, \mathrm{ii}, \mathrm{iii}, \mathrm{iv})]$ \\
\hline Hydrocotyle ranunculoides L. f. & VU & EN & \\
\hline Isoëtes histrix Bory & LR (LC) & & \\
\hline Limonium capitis-marci Arrigoni et Diana & EN & EN & $\mathrm{CR}[\mathrm{B} 1 \mathrm{ab}(\mathrm{i}, \mathrm{ii}, \mathrm{iii}, \mathrm{iv}, \mathrm{v})+2 \mathrm{ab}(\mathrm{i}, \mathrm{ii}, \mathrm{iii}, \mathrm{iv}, \mathrm{v})]$ \\
\hline Limonium cornusianum Arrigoni et Diana & & & $\mathrm{VU}[\mathrm{B} 1 \mathrm{ab}(\mathrm{i}, \mathrm{ii}, \mathrm{iii}, \mathrm{iv}, \mathrm{v})+2 \mathrm{ab}(\mathrm{i}, \mathrm{ii}, \mathrm{iii}, \mathrm{iv}, \mathrm{v})]$ \\
\hline Limonium lausianum Pignatti & VU & VU & CR [B1ab(i, ii, iii, iv, v) + 2ab(i, ii, iii, iv, v)] \\
\hline Limonium oristanum Mayer & & & $\mathrm{CR}[\mathrm{B} 1 \mathrm{ab}(\mathrm{i}, \mathrm{ii}, \mathrm{iii}, \mathrm{iv}, \mathrm{v})+2 \mathrm{ab}(\mathrm{i}, \mathrm{ii}, \mathrm{iii}, \mathrm{iv}, \mathrm{v})]$ \\
\hline Limonium pseudolaetum Arrigoni et Diana & VU & VU & $\mathrm{CR}[\mathrm{B} 1 \mathrm{ab}(\mathrm{i}, \mathrm{ii}, \mathrm{iii}, \mathrm{iv}, \mathrm{v})+2 \mathrm{ab}(\mathrm{i}, \mathrm{ii}, \mathrm{iii}, \mathrm{iv}, \mathrm{v})]$ \\
\hline Limonium tenuifolium (Bertol. ex Moris) Erben & LR (LC) & LR & DD \\
\hline Limonium tharrosianum Arrigoni et Diana & LR (LC) & LR & CR [B1ab(i, ii, iii, iv, v) + 2ab(i, ii, iii, iv, v)] \\
\hline Matthiola sinuata (L.) R. Br. & LR (LC) & & \\
\hline $\begin{array}{l}\text { Micromeria filiformis (Aiton) Benth. } \\
\text { subsp. filiformis }\end{array}$ & & & CR [B1ab(i, ii, iii, iv, v) + 2ab(i, ii, iii, iv, v)] \\
\hline Nananthea perpusilla (Loisel.) DC. & LR (LC) & LR & $\mathrm{EN}[\mathrm{B} 1 \mathrm{ab}(\mathrm{i}, \mathrm{ii}, \mathrm{iii}, \mathrm{iv}, \mathrm{v})+2 \mathrm{ab}(\mathrm{i}, \mathrm{ii}, \mathrm{iii}, \mathrm{iv}, \mathrm{v})]$ \\
\hline Narcissus tazetta L. & LR (LC) & & \\
\hline Nymphoides peltata (S.G. Gmel.) Kuntze & DD & EN & \\
\hline Pancratium maritimum $\mathrm{L}$. & LR (LC) & & \\
\hline Parapholis marginata Runemark & VU & & \\
\hline Pinus pinea $\mathrm{L}$. & LR (LC) & & \\
\hline Polygala sinisica Arrigoni & $\mathrm{CR}$ & $\mathrm{CR}$ & $\mathrm{CR}[\mathrm{Blab}(\mathrm{i}, \mathrm{ii}, \mathrm{ii}, \mathrm{iv}, \mathrm{v}) \mathrm{c}(\mathrm{iv})+2 \mathrm{ab}(\mathrm{i}, \mathrm{ii}, \mathrm{iii}, \mathrm{iv}, \mathrm{v}) \mathrm{c}(\mathrm{iv})+\mathrm{D}]$ \\
\hline Quercus calliprinos Webb & LR (LC) & & \\
\hline Ranunculus cordiger Viv. & & & \\
\hline subsp. diffusus (Moris) Arrigoni & CR. & $\mathrm{CR}$ & \\
\hline Romulea ligustica Parl. & & $\mathrm{CR}$ & \\
\hline Salicornia patula Duval-Jouve & LR (LC) & & \\
\hline $\begin{array}{l}\text { Silene succulenta Forssk. } \\
\text { subsp. corsica (DC.) Nyman }\end{array}$ & & VU & \\
\hline Scrophularia ramosissima Loisel. & LR (LC) & LR & \\
\hline $\begin{array}{l}\text { Teucrium subspinosum Pourr. ex Willd. } \\
\text { subsp. subspinosum }\end{array}$ & $\mathrm{LR}(\mathrm{LC})$ & LR & \\
\hline Urginea fugax (Moris) Steinh. & LR (LC) & VU & \\
\hline Vaccaria hispanica (Mill.) Rauschert & VU & & \\
\hline Viola arborescens $\mathrm{L}$. & VU & VU & \\
\hline
\end{tabular}

Tabella 4. Elenco taxa inseriti nelle liste rosse della IUCN e proposti per la tutela in Sardegna. List of Sardinian preservation taxa included in the IUCN red list. 
di più la creazione di un sottosettore biogeografico.

Per quanto concerne la tutela della diversità vegetale, si ritiene importante elaborare corrette strategie di conservazione, sia in situ che ex situ, per tutte le specie che rientrano in categorie a elevato grado di rischio o vulnerabilità, quali Polygala sinisica, Viola arborescens, Helianthemum caput-felis, Coris monspeliensis subsp. monspeliensis, Ferula arrigonii, Nananthea perpusilla, Ephedra distachya, Armeria pungens, Delphinium longipes e per le specie endemiche del genere Limonium. Queste misure di tutela sarebbero peraltro in linea con gli strumenti già previsti, quali il parco regionale "Sinis-Montiferru" (Legge Regionale 31/89), l'Area Marina Protetta "Penisola del Sinis-Isola di Mal di Ventre" e la presenza di ben 8 proposti Siti di Importanza Comunitaria, 4 Zone di Protezione Speciale per gli uccelli istituite e una quinta in corso di istituzione della rete Natura 2000.

RINGRAZIAMENTI. Un ringraziamento all'Area Marina Protetta "Penisola del Sinis. Isola di Mal di Ventre", e in particolare al direttore Bruno Paliaga, per aver promosso tale lavoro e messo a disposizione i dati in possesso; un ringraziamento particolare a Simona Pallanza, Giorgio Massaro e Luca Loria per l'aiuto nella raccolta dei dati e la partecipazione alle escursioni.

\section{BIBLIOGRAFIA}

ANGIOLINI, C., F. FRIGNANI \& M. LANDI 2004- Alcune note su Ononis viscosa L. e O. breviflora DC. in Italia. Inform. Bot. Ital. 36(1): 168-174.

ANGIOSPERM PHYLOGENY GROUP -2003An update of the Angiosperm Phylogeny Group classification for the orders and families of flowering plants: APG II. Bot. J. Linn. Soc. 141(4): 399-436.
ARRIGONI, P.V. -1971- Helianthemum caputfelis Boiss. $(2 \mathrm{n}=24)$ nuovo reperto per la flora italiana. Webbia 26(1): 237-243.

ARRIGONI, P.V. -1972- Nuovi reperti di alcune specie rare o notevoli della flora sarda. Webbia 27(1): 273-279.

ARRIGONI, P.V. -1986- Le piante endemiche della Sardegna: 181. Boll. Soc. Sarda Sci. Nat. 25: 159-163.

ARRIGONI, P.V. \& P.L. DI TOMMASO -1991La vegetazione delle montagne calcaree della Sardegna centro-orientale. Boll. Soc. Sarda Sci. Nat. 28: 201-310.

ARRIGONI, P.V. \& S. DIANA -1999- Karyology, chorology and bioecology of the genus Limonium (Plumbaginaceae) in Sardinia. Plant Biosystems 133(1): 63-71.

ARRIGONI, P.V., I. CAMARDA, B. CORRIAS, S. DIANA CORRIAS, E. NARDI, M. RAFFAELLI \& F. VALSECCHI -1976/1977/ 1991- Le piante endemiche della Sardegna: 1-202. Boll. Soc. Sarda Sci. Nat. 16-28.

ATZEI A.D. -1981- Segnalazioni floristiche italiane:53. Inform. Bot. Ital. 12(1): 71.

BACCHETTA G. -2006- Flora vascolare del Sulcis (Sardegna Sud-Occidentale, Italia). Guineana 12: 1-350.

BACCHETTA, G. \& C. PONTECORVO -2005Contribution to the knowledge of the endemic vascular flora of Iglesiente (SW SardiniaItaly). Candollea 60(2): 481-501.

BACCHETTA, G., A. COPPI, C. PONTECORVO \& F. SELVI -2007- Systematics, phylogenetic relationships and conservation of the taxa of Anchusa (Boraginaceae) endemic to Sardinia (Italy). Systematic and Biodiversity (in press).

BAGELLA, S. \& M. URBANI -2006- Vascular flora of the calcareus outcrops in NorthWestern Sardinia (Italy). Webbia 61(1): 95132.

BANFI, E., G. GALASSO \& A. SOLDANO 2005- Notes on systematics and taxonomy for the Italian vascular flora. 1. Atti Soc. It. Sci. Nat. 146 (II): 219-244.

BARBEY, W. -1885- Florae sardoae compendium. Ed. Bridel, Lausanne.

BATEMAN, R.M., P. M. HOLLINGSWORTH, J. PRESTON, L. YI-BO, A.M. PRIDGEON \& M.W. CHASE -2003- Molecular phylogenetics and evolution of Orchidinae 
and selected Habenariinae (Orchidaceae). Bot. J. Linn. Soc. 142: 1-40.

BOCCHIERI, E. -1996- L'esplorazione botanica e la principali conoscenze sulla flora dell'arcipelago della Maddalena (Sardegna nord-orientale). Rend. Sem. Fac. Sci. Univ. Cagliari 66(suppl.): 1-305.

BOCCHIERI, E. \& B. MULAS -1996Phytogeographic studies in the Sinis peninsula (C.W. Sardinia): Capo S. Marco. Flora Mediterranea 6: 119-147.

BOCCHIERI, E., B. MULAS \& G. AVENA 1988- La flora della Penisola di Capo Mannu (Sardegna centro-occidentale). Webbia 42(2): 201-225.

BOLÒS, O. \& J. VIGO -1984/2001- Flora dels Països Catalans, 1-4. Editorial Barcino, Barcelona.

BRAMBILLA, C., G. CANEVA, G. DE MARCO \& L. MOSSA -1982- Analisi fitosociologica della seriazione psammofila costiera nella Sardegna Meridionale. Ann. Bot. (Roma) 40: 69-96.

BRULLO S., M. GRILLO \& A. GUGLIELMO 1996- Considerazioni fitogeografiche sulla flora iblea. Boll. Acc. Gioenia Sci. Nat. 29: 45-111.

BRUMMITT, R.K. \& C.E. POWELL (Eds.) 1992-Authors of plant names. Royal Botanic Gardens, Kew.

CARDONA, M.A. \& E. SIERRA-RÀFOLS 1981- Contribución al estudio del genero Rubia. I. Táxones mediterráneo-occidentales y macaronésicos. Anales Jard. Bot. Madrid 37(2): 557-575.

CASTROVIEJO, S. (Ed.) -1986/2006- Flora iberica. Plantas vasculares de la Peninsula Ibérica e Islas Baleares. 1-8, 10, 14, 21. Real Jardin Botànico. C.S.I.C, Madrid.

CHERCHI, A., A. MARINI, M. MURRU \& E. ROBBA -1978- Stratigrafia e paleoecologia del Miocene Superiore della Penisola del Sinis (Sardegna Occidentale). Riv. Ital. Paleont. 84(4): 973-1036.

CONTI, F., A. MANZI \& F. PEDROTTI -1997Liste rosse regionali delle piante d'Italia. WWF, Società Botanica Italiana, Camerino.

CONTI, F., G. ABBATE, A. ALESSANDRINI \& C. BLASI- 2005-An Annotated Checklist of the Italian Vascular Flora. Palombi Editore,
Roma.

CORRIAS, B. -1977- Ad floram italicam notulae taxonomicae et geobotanicae. 21. Nananthea perpusilla (Loisel.) DC. Webbia 31(1): 43-48.

CORRIAS, B. -1981- Le piante endemiche della Sardegna: 93. Boll. Soc. Sarda Sci. Nat. 20: 282-286

DANIN, A., G. DOMINA \& F.M. RAIMONDO -2005- Prime osservazioni ecologicodistributive su Arundo collina Ten. (Poaceae) in Italia. Inform. Bot. Ital. 37(2): 1167-1170.

DELFORGE, P. -2005- Guide des Orchidées d'Europe, d'Afrique du Nord et du ProcheOrient. $3^{\circ}$ édition. Delachaux et Niestlé, Paris.

DERIU, M. \& M. ZERBI -1964- Notizie sulla costituzione geopetrografica dell'Isola di Mal di Ventre. Ateneo Parm., Acta Nat. 35: 113143.

DESOLE, L. -1956- Nuove stazioni di Coeloglossum diphyllum Fiori in Sardegna Isola madre. Nuovo Giorn. Bot. Ital 63: 257282.

DESOLE, L. -1961- La vegetazione. In Ricerche sull'Arcipelago de La Maddalena. Mem. Soc. Geogr. Ital. 25: 89-186.

DESOLE, L. -1965- Distribuzione geografica del genere Ephedra in Sardegna: $3^{\circ}$ nota. Ephedra distachya L. (dal Golfo di Oristano all'Arcipelago della Maddalena). Bull. Ist. Bot. Univ. Sassari 7(3): 3-57.

ENEA -1990- Indagine sulla situazione ambientale delle aree destinate a riserve marine: "Penisola del Sinis- Isola di Mal di Ventre". La Spezia.

FORTI, S. \& P. ORRU -1995- Geomorfologia costiera e sottomarina della Penisola del Sinis (Sardegna Occidentale). Boll. Soc. Geol. It. 114: 3-21.

GAMISANS, J. \& J.F. MARZOCCHI -1996- La flore endémique de la Corse. Edisud, Aix en Provence.

GENNARI, P. -1870- Florula di Caprera. Nuovo Giorn. Bot. Ital. 2: 90-145.

GIOTTA C. \& M. PICCITTO -1995- Neue Angaben zur verbreitung einiger Sardischer Orchideen. Journ. Eur. Orch. 27(2): 285-295.

GREUTER, W., H.M. BURDET \& G. LONG (Eds.) -1984/89-Med-Checklist, 1, 2, 4. Conserv. et Jard. Bot. Genève, Genève. 
IUCN -1994- IUCN Red List Categories. IUCN Species Survival Commission. IUCN, Gland and Cambridge.

IUCN -2001- IUCN Red List Categories and Criteria: Version 3.1. IUCN Species Survival Commission. IUCN, Gland and Cambridge.

IUCN- 2006- Guidelines for using IUCN Red List Categories and Criteria. IUCN-SSC, Gland, Switzerland and Cambridge, UK.

KERGUÉLEN, M. -1987- Données taxonomiques, nomenclaturales et chorologiques pour une révision de la flore de France. Lejeunia, Nouv. Sér. 120: 263.

LADERO ALVAREZ, M., T.E. DÍAZ GONZALEZ, A. PENAS MERINO, S. RIVAS-MARTÍNEZ \& C. VALLE GUTIÉRREZ -1987- Datos sobre la vegetación de las Cordilleras Central y Cantábrica. Itin. Geobot. 1: 3-147.

MARCHETTI, D.- 2004- Le Pteridofite d'Italia. Ann. Mus. civ. Rovereto, Sez.: Arch. St., Sc., nat. 19(2003): 71-231.

MAYER, A. -1995-Comparative study of the coastal vegetation of Sardinia (Italy) and Crete (Greece) with respect to the effects of human influence. IHW-Verlag (Libri Botanici, vol 15), Munchen.

MÉDAIL, F. \& P. QUÉZEL -1997- Hot-spots analysis for conservation of plant biodiversity in the mediterranean basin. Mediterranean plant biodiversity 84(1): 112-127.

MONTMOLLIN DE, B. \& W. STRAHM (Eds.)2005- The Top 50 Mediterranean Island Plants: wild plants at the brink of extinction, and what is needed to save them. IUCN/SSC Mediterranean Islands Plant Specialist Group. IUCN, Gland, Switzerland and Cambridge, UK.

MOSSA, L. -1990- La vegetazione forestale del campo dunale di Bugerru-Portixeddu (Sardegna occidentale). Ann. Bot., Studi sul Territorio 48(7): 291-306.

MOSSA, L., A. SCRUGLI \& G. MILIA -1984Flora e vegetazione dell'Isola di Mal di Ventre (Sardegna centro-occidentale). Rend. Sem. Fac. Sci. Univ. Cagliari 54: 119-142.

MULAS, B. -1984- Micromeria filiformis (Aiton) Bentham in Sardegna, specie nuova per la flora d'Italia. Webbia 37(2): 237-242.

MULAS, B. -1986- La flora dello stagno di "Mari
Ermi" (Cabras, Sardegna centro-occidentale). Rend. Sem. Fac. Sci. Univ. Cagliari 56(2): $27-$ 41.

MULAS, B. -1993- La flora del promontorio di Torre del Sevo (Sardegna CentroOccidentale). Webbia 47(2): 259-276.

NICOTRA, L. -1898- La Viola arborescens L. nella Flora d'Italia. Malpighia 12: 238-240.

PIGNATTI, S. -1982- Flora d'Italia, 1-3. Edagricole, Bologna.

PIGNATTI, S., P. MENEGONI \& V. GIACANELLI (Eds.)- 2001- Liste rosse e blu della flora italiana. ANPA, Roma.

PYSEK, P., D.M. RICHARDSON, M REJMÁNEK, G.L. WEBSTER, M. WILLIAMSON \& J. KIRSCHNER- 2004Alien plants in checklists and floras: towards better communication between taxonomists and ecologist. Taxon 53(1): 131-143.

RABINOWITZ, D. -1981- Seven forms of rarity. In SYNGE H. (Ed.)- The biological aspects of rare plants conservation. Chichester, U.K.

RABINOWITZ, D., S. CAIRNS \& T. DILLON 1986- Seven forms of rarity and their frequency in the flora of the British Isles. In SOULE, M.E. (Ed.)- Conservation Biology: the Science of Scarcity and Diversity. Sunderland, Massachusetts.

RAUNKIAER, C. -1934- The life forma of plants and statistical plant geography. Univ. Oxford, Oxford.

RIVAS-MARTÍNEZ, S. -1996- Clasificación bioclimática de la tierra. Folia Botanica Matritensis 17: 1-32.

RIVAS-MARTÍNEZ, S., D. SANCHEZ-MATA \& M. COSTA -1999- North American boreal and western temperate forest vegetation (Syntaxonomical synopsis of the potential natural plant communities of North America, II). Itin. Geobot. 12: 5-316.

RIVAS-MARTÍNEZ， S., T.E. DÍAZ，F. FERNÁNDEZ-GONZÁLEZ, J. IZCO, J. LOIDI, M. LOUSÃ \& A. PENAS- 2002Vascular plant communities of Spain and Portugal. Itin. Geobot. 15(1): 5-432.

SCOPPOLA, A. \& G. SPAMPINATO (Eds.)2005-Atlante delle specie a rischio di estinzione. In SCOPPOLA, A. \& C. BLASI (Eds.) (2005) Stato delle conoscenze sulla flora vascolare d'Italia. Palombi Editore, 
Roma.

SCRUGLI, A. \& A. COGONI -1995- Primo reperto di Gennaria diphylla Parl. per la Sardegna meridionale. Giorn. Bot. Ital. 129(2): 185.

TUTIN, T.G., V.H. HEYWOOD, N.A. BURGES, D.M. MOORE, D.H. VALENTINE, S.M. WALTERS \& D.A. WEBB -1993- Flora Europaea, 1. Cambridge University Press, Cambridge.

TUTIN. T.G., N.A. BURGES, A.O. CHATER, J.R. EDMONDSON, V.H. HEYWOOD, D.M. MOORE, D.H. VALENTINE, S.M. WALTERS \& D.A. WEBB -1964/1980- Flora Europaea, 1-2-3-4-5. Cambridge University Press, Cambridge.
VACCARI, A. -1894- Flora dell'Arcipelago di Maddalena (Sardegna). Malpighia 8: 227277.

VACCARI, A. -1896- Supplemento alla Flora dell'Arcipelago di Maddalena. Malpighia 10: 521-534.

VALSECCHI, F. -1980- Le piante endemiche della Sardegna: 80. Boll. Soc. Sarda Sci. Nat. 19: 323-326.

VALSECCHI, F. -1981- Segnalazioni floristiche italiane: 46. Inform. Bot. Ital. 11(3): 327.

VALSECCHI, F. -1995- Indagini sistematiche, tassonomiche e corologiche nel gruppo "Silene colorata Poir. S. sericea All. S. canescens Ten.”. Boll. Soc. Sarda Sci. Nat. 30: 447-476. 